Supporting Information for

\title{
A Covalent Organic Framework Bearing Single Ni Sites as a Synergistic Photocatalyst for Selective Photoreduction of $\mathrm{CO}_{2}$ to $\mathrm{CO}$
}

Wanfu Zhong ${ }^{\dagger, \#}$, Rongjian Sa ${ }^{\ddagger}, \#$, Liuyi Li ${ }^{\dagger, *}$, Yajun $\mathrm{He}^{\dagger}$, Lingyun $\mathrm{Li}^{\dagger}$, Jinhong Bi ${ }^{\dagger}$, Zanyong Zhuang ${ }^{\dagger}$, Yan $\mathrm{Yu}^{\dagger, *}$, Zhigang Zou ${ }^{\dagger, \S, *}$

$\dagger$ Key Laboratory of Eco-materials Advanced Technology, College of Materials Science and Engineering, Fuzhou University, Fuzhou 350108, China.

† Institute of Oceanography, Ocean College, Minjiang University, Fuzhou, Fujian 350108, China.

$\S$ Eco-materials and Renewable Energy Research Center, College of Engineering and Applied Sciences, Nanjing University, Nanjing 210093, China.

\section{Table of Contents}

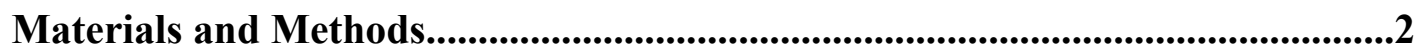

Computational details.......................................................................................2

Supplementary Figures.....................................................3

Calculated Geometries Parameters.........................................12

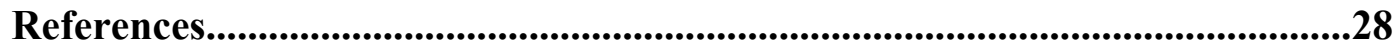




\section{Materials and Methods}

5,5'-diamino-2,2'-bipyridine ${ }^{[1]}$ and triformylphloroglucinol ${ }^{[2]}$ were synthesized according to literature methods. Other starting materials were commercially available and used without further purification. ${ }^{1}$ H NMR spectra was recorded on a Bruker AVANCE III NMR spectrometer at 400 $\mathrm{MHz}$, respectively, using tetramethylsilane (TMS) as an internal standard. Solid-state ${ }^{13} \mathrm{C}$ CP/MAS NMR was performed on a Bruker SB Avance III $500 \mathrm{MHz}$ spectrometer with a 4-mm double-resonance MAS probe. FTIR spectra were recorded with KBr pellets using Perkin-Elmer Instrument. Powder X-ray diffraction (XRD) patterns were recorded in the range of $2 \theta=3-40^{\circ}$ on a desktop X-ray diffractometer (RIGAKU-Miniflex II) with $\mathrm{Cu} \mathrm{K \alpha}$ radiation $\left(\lambda=1.5406 \AA\right.$ ). $\mathrm{N}_{2}$ or $\mathrm{CO}_{2}$ adsorption and desorption isotherms were measured at $77 \mathrm{~K}$ using a Micromeritics ASAP 2020 system. The samples were degassed at $120{ }^{\circ} \mathrm{C}$ for $10 \mathrm{~h}$ before the measurements. Surface areas were calculated from the adsorption data using Brunauer-Emmett-Teller (BET) equation. The calculation of the pore size distribution was done using the density functional theory (DFT) equilibrium model. Field-emission scanning electron microscopy (SEM) was performed on a JEOL JSM-7500F operated at an accelerating voltage of $3.0 \mathrm{kV}$. Transmission electron microscope (TEM) was obtained with TECNAI G ${ }^{2}$ F20. X-ray photoelectron spectroscopy (XPS) measurements were performed on a Thermo ESCALAB 250 spectrometer, using non-monochromatic $\mathrm{Al} \mathrm{K \alpha}$ x-rays as the excitation source and choosing $\mathrm{C} 1 \mathrm{~s}(284.8 \mathrm{eV})$ as the reference line. The concentration of $\mathrm{Ni}$ was analyzed by inductively coupled plasma optical emission spectrometry (ICP-OES; iCAP 7000, Thermo Fisher Scientific Inc.). Gas products are detected using gas chromatography (Agilent Technologies-7890B). UV-vis spectra were recorded using a Agilent Cary 5000 spectrometer. The number of incident photons was estimated by a calibrated Si photodiode, and it was supposed that all incident photons were absorbed by the photocatalyst.

\section{Computational details}

DFT calculations with the Grimme's D3 correction was applied in our calculations. The COF model was initial constructed and optimized based on PBE0-D3/def2-SVP level with all the aromatic ring keep vertical and the distances of neighboring layers fixed to $3.503 \AA$. The geometry of COF participant complex was optimized based on PBE0-D3/def2-SVP with COF fragment fixed. All the other investigated geometries were optimized at the level of PBE0-D3/def2-SVP without any constrains applied. Vibrational frequency analyses at the same basis sets were used on all optimized structures in order to characterize stationary points as local minima. The Gibbs free energies were obtained by a single-point energy calculations at the level of PBE0-D3/def2-TZVP based on the PBE-D3/def2-SVP optimized structures and thermal corrections. The Gaussian $09^{[3]}$ package was used for all of our calculations. 
Table S1. Constrained the optimized building block of TpBpy based complex model based on PBE0-D3/def2-svp level.

\begin{tabular}{lllll}
\hline & $\mathrm{M}-\mathrm{C}(\AA)$ & $\mathrm{C}-\mathrm{O}(\AA)$ & $\mathrm{C}-\mathrm{O}(\AA)$ & O-C-O (deg) \\
Free $\mathrm{CO}_{2}$ & & 1.160 & 1.160 & 180.0 \\
{$\left[\mathrm{~L}_{2} \mathrm{Ni}-\mathrm{CO}_{2}\right]$} & 3.069 & 1.161 & 1.161 & 176.1 \\
{$\left[\mathrm{~L}_{2} \mathrm{Ni}-\mathrm{CO}_{2} \mathrm{H}\right]^{+}$} & 1.881 & 1.347 & 1.210 & 120.5 \\
{$\left[\mathrm{COF}-\mathrm{Ni}-\mathrm{CO}_{2}\right]$} & 1.869 & 1.223 & 1.229 & 138.2 \\
{$\left[\mathrm{COF}-\mathrm{Ni}-\mathrm{CO}_{2} \mathrm{H}\right]^{+}$} & 1.866 & 1.314 & 1.221 & 124.1 \\
Free $\mathrm{H}_{2} \mathrm{O}$ & & $0.963^{a}$ & $0.963^{a}$ & $103.2^{b}$ \\
{$\left[\mathrm{~L}_{2} \mathrm{Ni}-\mathrm{H}_{2} \mathrm{O}\right]$} & $3.141^{c}$ & $0.971^{a}$ & $0.974^{a}$ & $103.9^{b}$ \\
{$\left[\mathrm{~L}_{2} \mathrm{Co}-\mathrm{CO}_{2}\right]^{+}$} & 3.150 & 1.160 & 1.161 & 179.0 \\
{$\left[\mathrm{~L}_{2} \mathrm{Co}-\mathrm{COFCO}_{2}\right]^{+}$} & 1.879 & 1.237 & 1.200 & 145.4 \\
{$\left[\mathrm{~L}_{2} \mathrm{Co}-\mathrm{H}_{2} \mathrm{O}\right]^{+}$} & $3.276^{d}$ & $0.963^{a}$ & $0.969^{a}$ & $104.3^{b}$ \\
\hline
\end{tabular}

${ }^{a}$ The bond length of $\mathrm{H}-\mathrm{O} ;{ }^{b}$ The angle of H-O-H in degree; ${ }^{c}$ The bond length of Ni-O; ${ }^{d}$ The bond length of Co-O.

\section{Supplementary Figures}

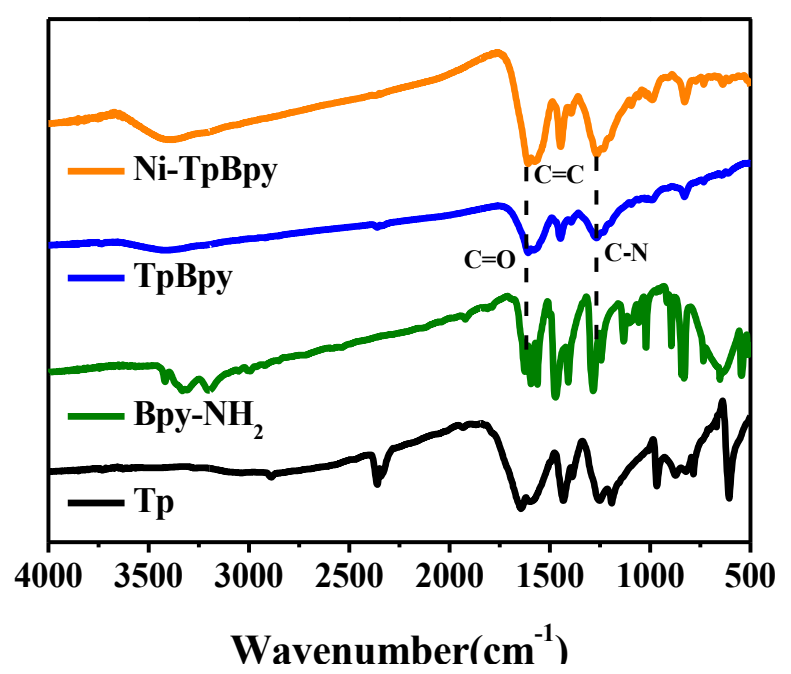

Figure S1. FTIR spectra of Tp, Bpy- $\mathrm{NH}_{2}$, TpBpy and Ni-TpBpy. Tp and $\mathrm{Bpy}-\mathrm{NH}_{2}$ are triformylphloroglucinol and 5,5'-diamino-2,2'-bipyridine, respectively. 


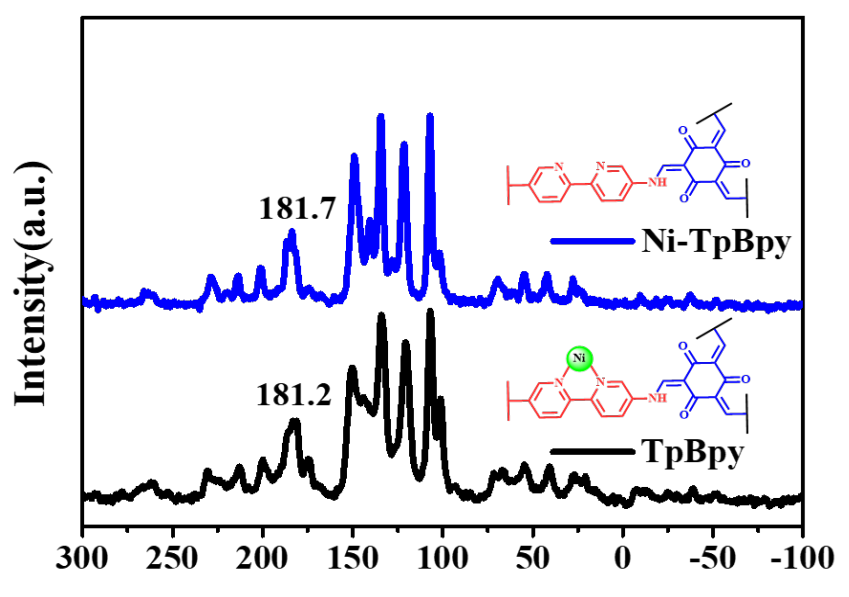

\section{Chemical Shift (in ppm)}

Figure S2. Solid-state ${ }^{13} \mathrm{C}$ NMR spectra of TpBpy and Ni-TpBpy.

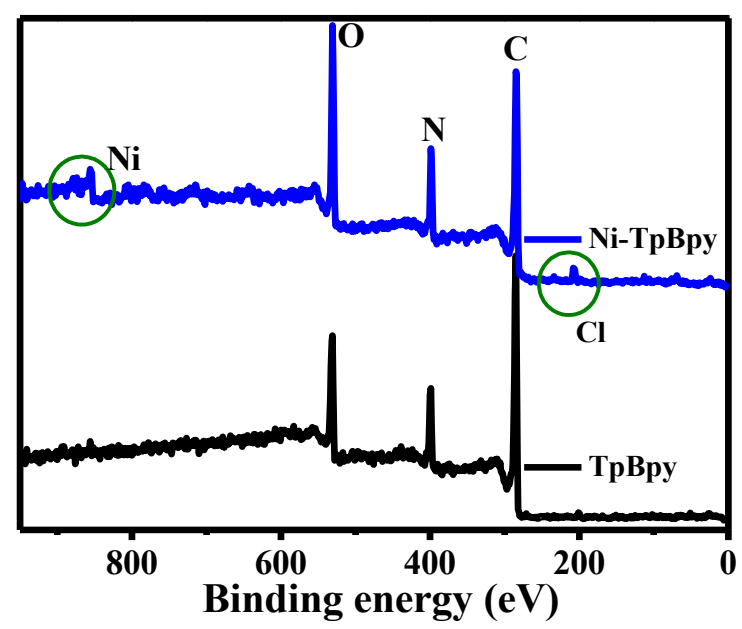

Figure S3. Survey scan XPS profile of TpBpy and Ni-TpBpy.
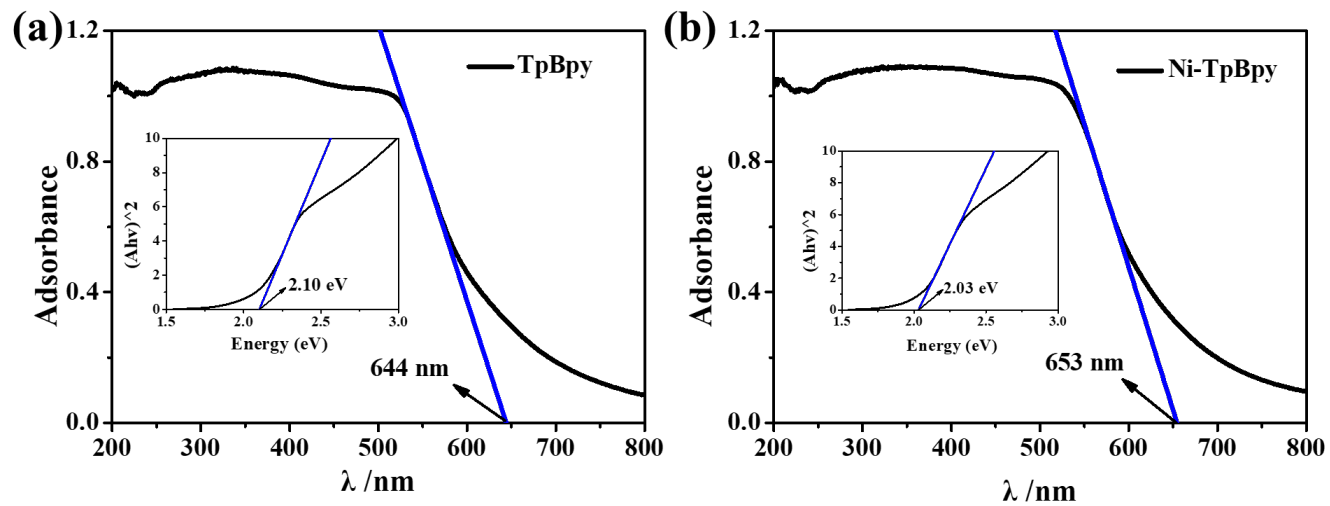

Figure S4. The UV-vis diffuse reflectance spectra and band gap energy of TpBpy (a) and Ni-TpBpy (b). 


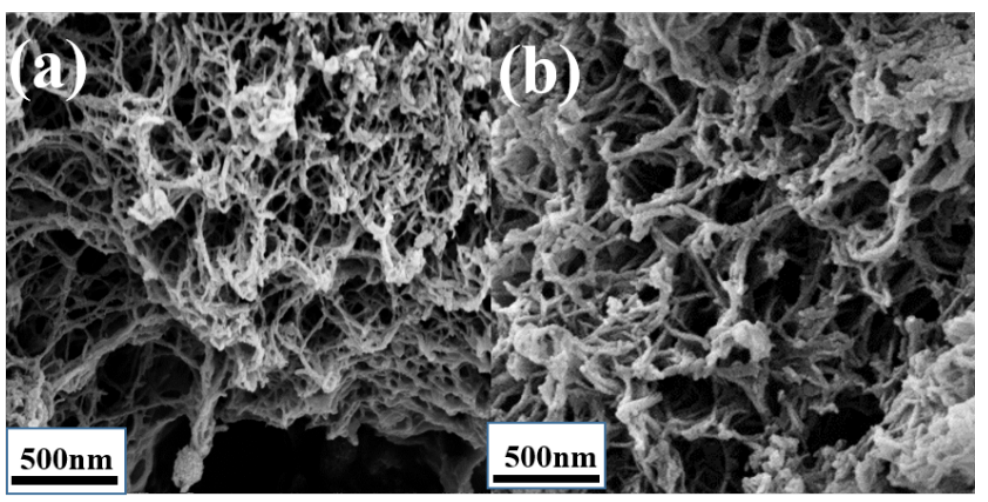

Figure S5. SEM images of TpBpy (a) and Ni-TpBpy (b).

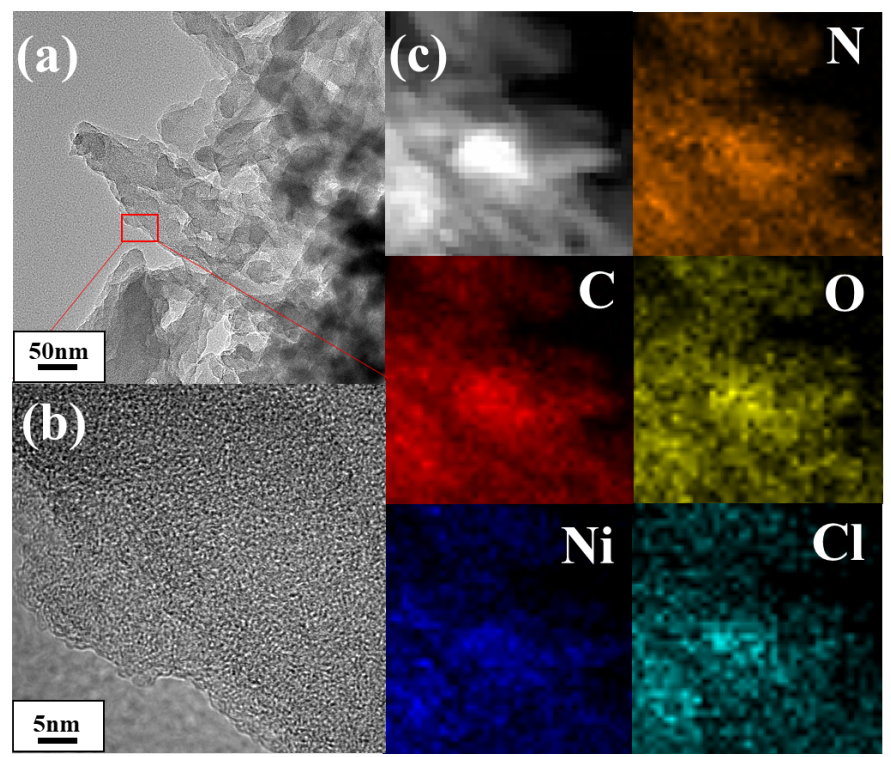

Figure S6. EDX mapping of Ni-TpBpy.

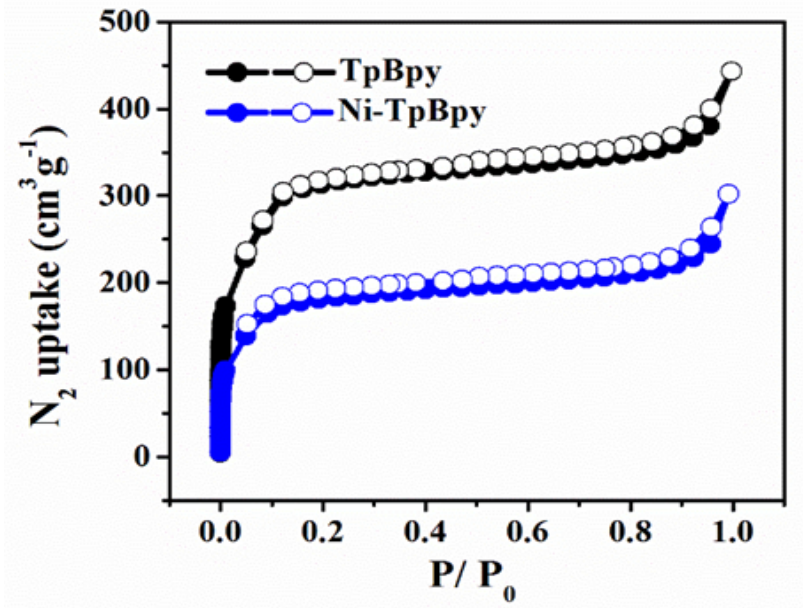

Figure S7. $\mathrm{N}_{2}$ sorption isotherms of the TpBpy and Ni-TpBpy. 


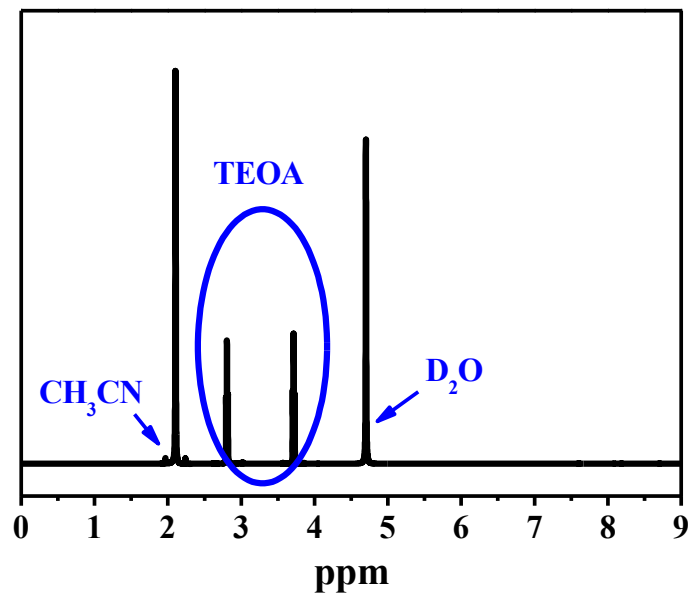

Figure S8. ${ }^{1} \mathrm{H}$ NMR spectrum of the liquid phase from the reaction system after visible-light irradiation for $4 \mathrm{~h}$.

No liguid hydrocarbon products derived from $\mathrm{CO}_{2}$ such as $\mathrm{CH}_{3} \mathrm{OH}, \mathrm{HCHO}$ and $\mathrm{HCOOH}$ are detected through analyzing the solvent after photocatalytic reactions by ${ }^{1} \mathrm{H}-\mathrm{NMR}$ spectroscopy.

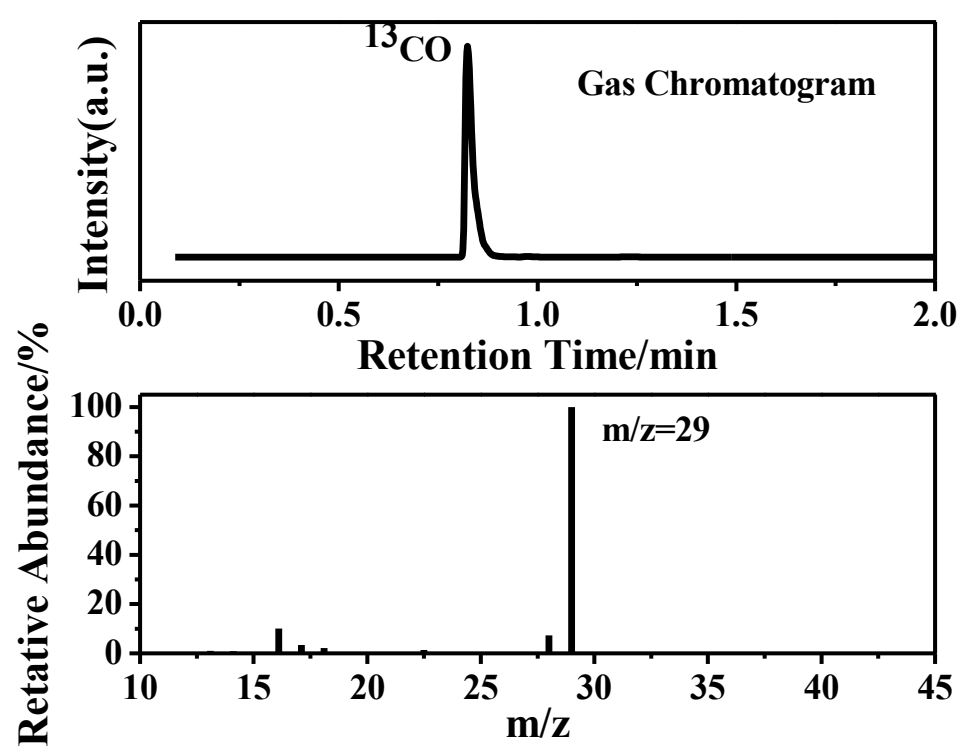

Figure S9. Gas chromatogram and mass spectra $(\mathrm{m} / \mathrm{z}=29)$ analyses of the carbon source of the generated $\mathrm{CO}$ from the Ni-TpBpy promoted photocatalytic ${ }^{13} \mathrm{CO}_{2}$ reduction system. 


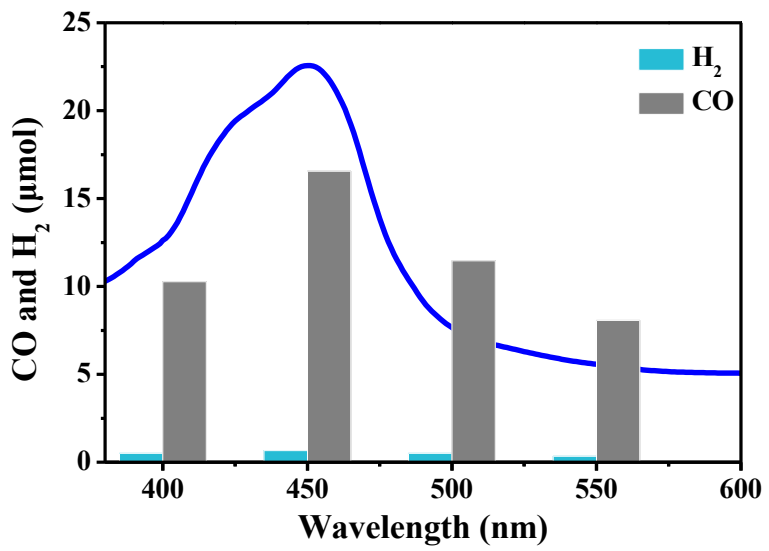

Figure S10. Wavelength dependence of production of $\mathrm{CO}$ and $\mathrm{H}_{2}$ in $4 \mathrm{~h}$ reaction.

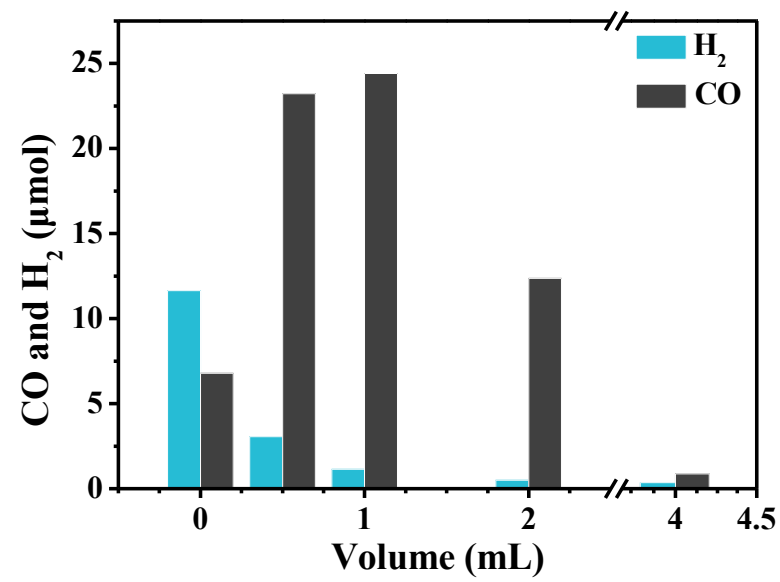

Figure S11. Water content during reaction dependence of production of $\mathrm{CO}$ and $\mathrm{H}_{2}$ in $2 \mathrm{~h}$ reaction.

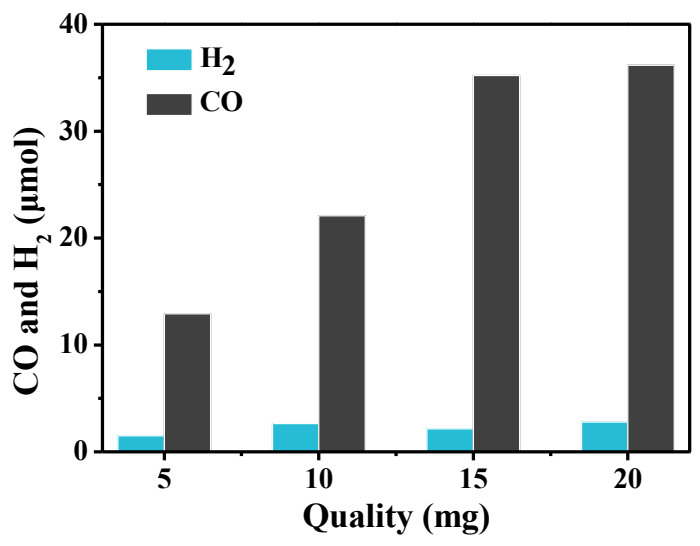

Figure S12. The effect of the amount of 2,2'-bipyridine on the evolution of $\mathrm{CO}$ and $\mathrm{H}_{2}$ from the $\mathrm{CO}_{2}$ photoreduction system in $4 \mathrm{~h}$ reaction.

The amount of 2,2'-bipyridine also significantly influences on the photocatalytic activity and selectivity (Fig.S11). Increasing the amount of 2,2'-bipyridine from 5 to $15 \mathrm{mg}$, the production of 
$\mathrm{H}_{2}$ increase slightly, while the production of $\mathrm{CO}$ increases obviously. Excessive 2,2'-bipyridine leads to a slight increase in $\mathrm{CO}$ generation.

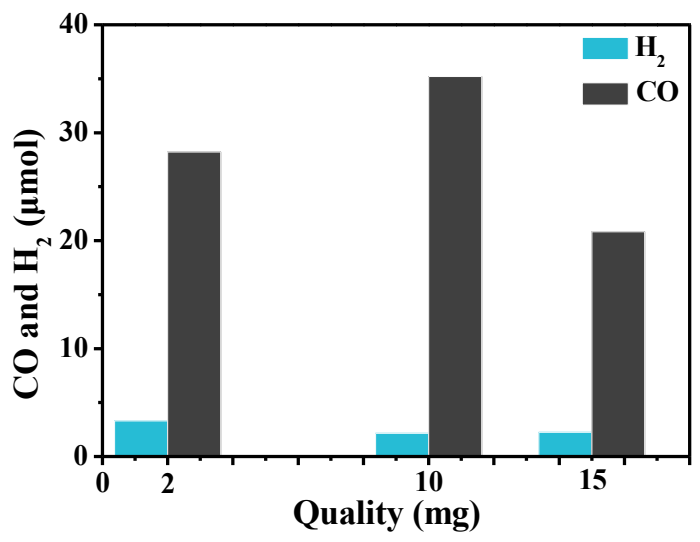

Figure S13. The effect of the amount of Ni-TpBpy on the evolution of $\mathrm{CO}$ and $\mathrm{H}_{2}$ from the $\mathrm{CO}_{2}$ photoreduction system in a $4 \mathrm{~h}$ reaction.

When $10 \mathrm{mg}$ Ni-TpBpy was added to the reaction system, the activity reached a maximum value for the production of CO. Further increasing the amount of Ni-TpBpy, the amount of CO decreases mainly because of the light shielding effect in the reaction.

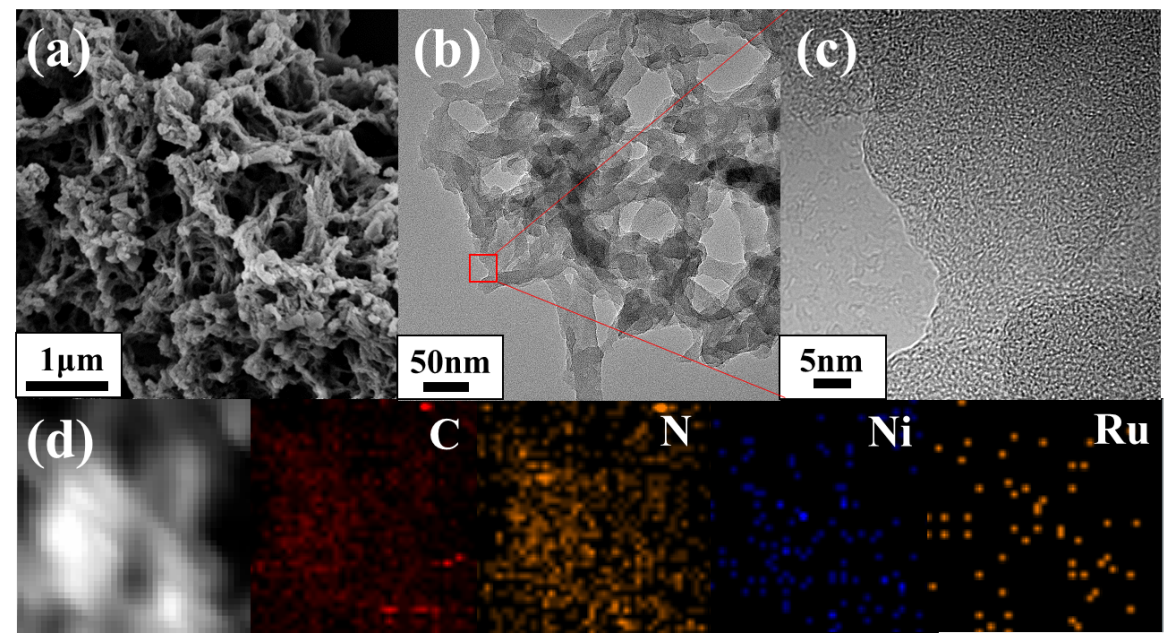

Figure S14. SEM(a); TEM (b-c) and (d) EDX elemental mapping of Ni-TpBpy after catalytic reaction. 


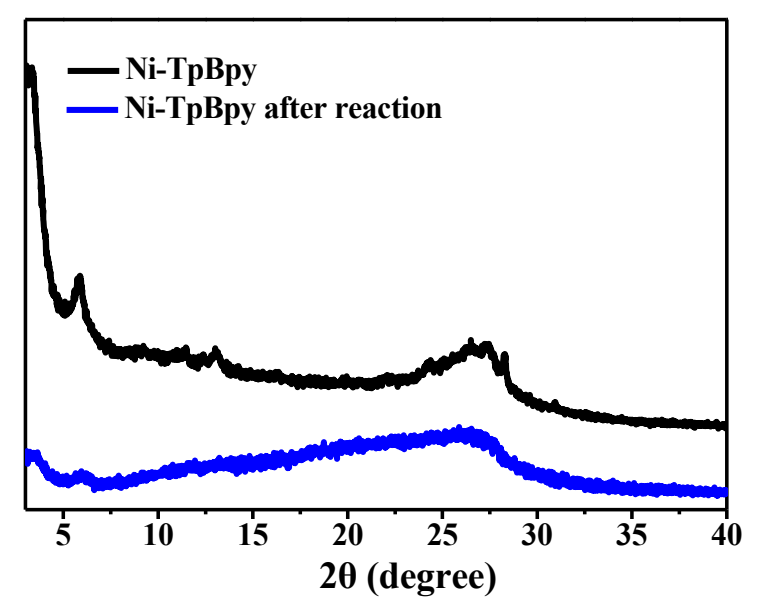

Figure S15. PXRD patterns of Ni-TpBpy before and after reaction.

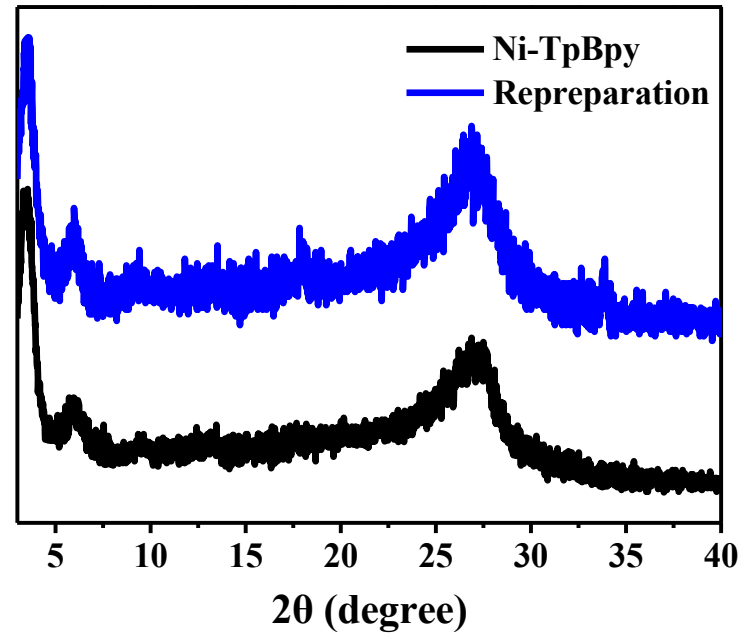

Figure S16. PXRD patterns of Ni-TpBpy (blue) and Ni-TpBpy after repreparation.

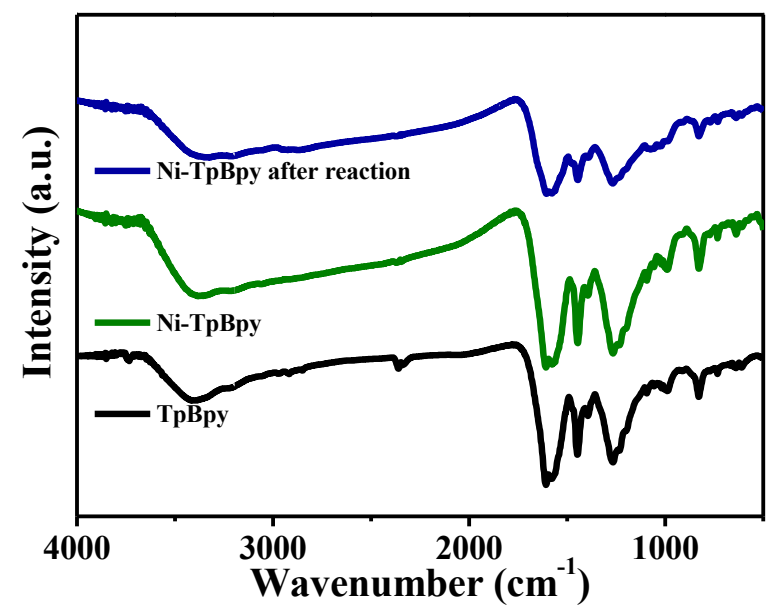

Figure S17. FTIR spectra of TpBpy, Ni-TpBpy and the recovered Ni-TpBpy after reaction. In the FTIR spectra of Ni-TpBpy at $1645 \mathrm{~cm}^{-1}(-\mathrm{C}=\mathrm{O}), 1593 \mathrm{~cm}^{-1}(-\mathrm{C}=\mathrm{C})$, and $1251 \mathrm{~cm}^{-1}(-\mathrm{C}-\mathrm{N})$ indicates the retention of keto-enol tautomerized structure of TpBpy. 


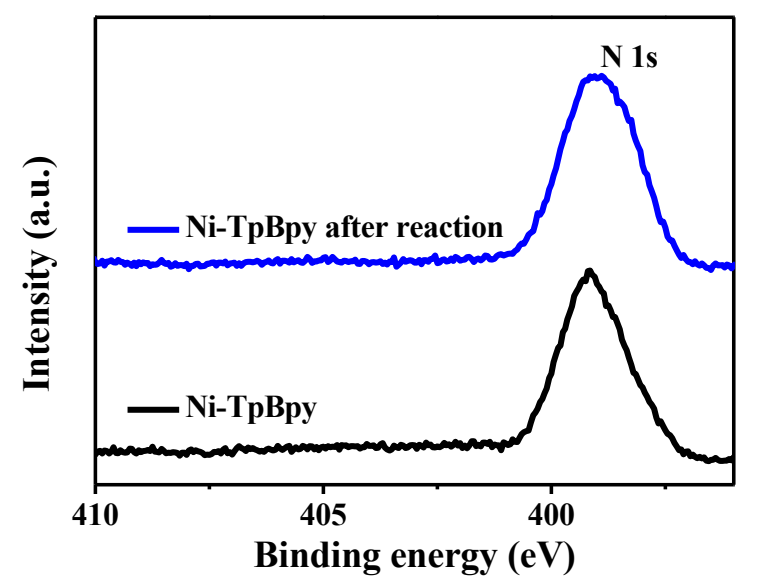

Figure S18. N 1s XPS spectra of Ni-TpBpy and Ni-TpBpy after reaction.

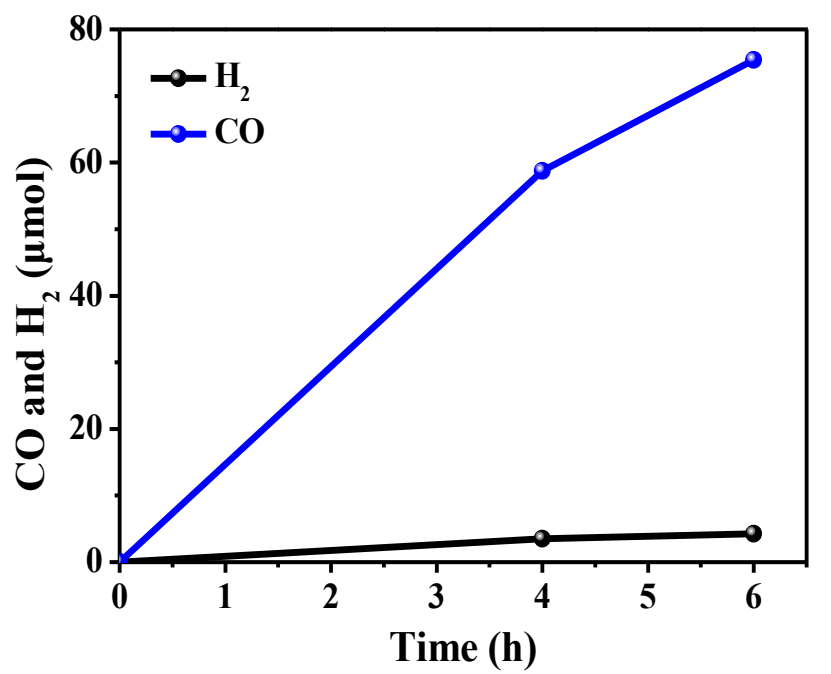

Figure S19. Reloading Ni in the recovered Ni-TpBpy for selective photoreduction of CO.

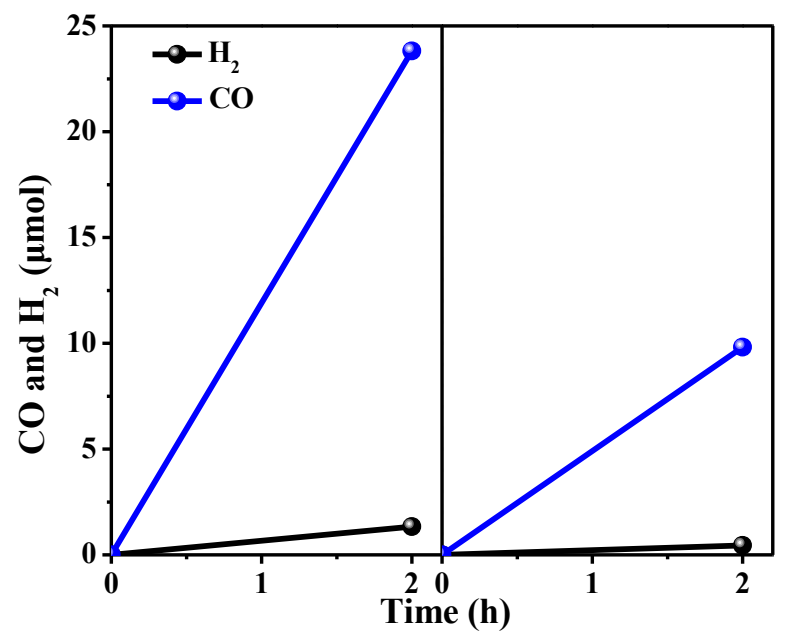

Figure S20. Filtration test for selective photoreduction of CO. (left) Two hour reaction, (right) after two hour of reaction, the reaction filtrate was filtered off and the filtrate was put back into the reaction. 

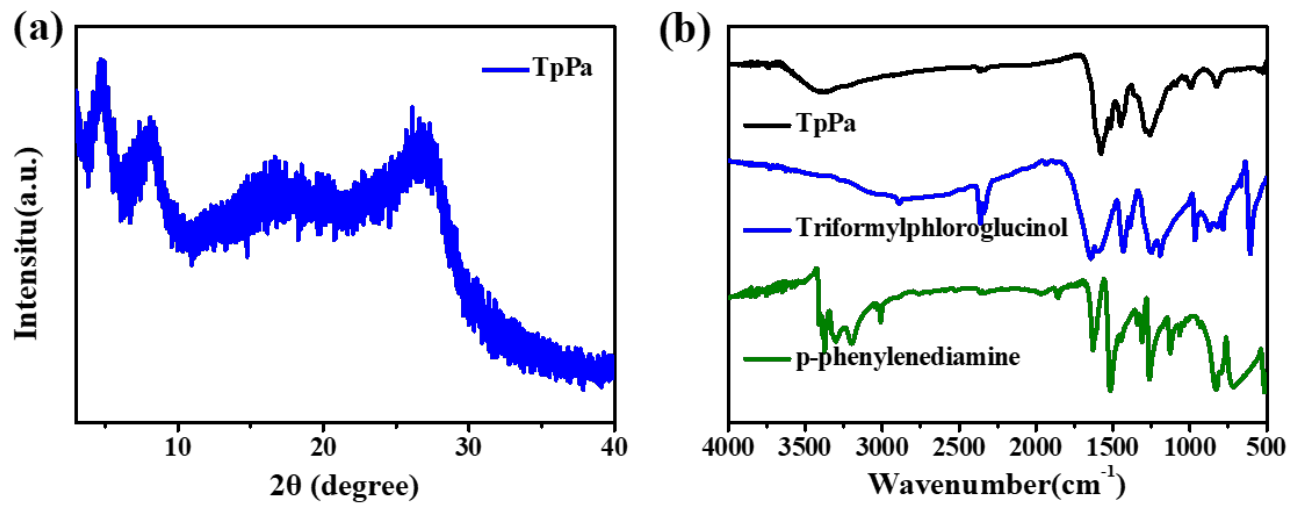

Figure S21. PXRD patterns (a) and FTIR spectra (b) of TpPa.
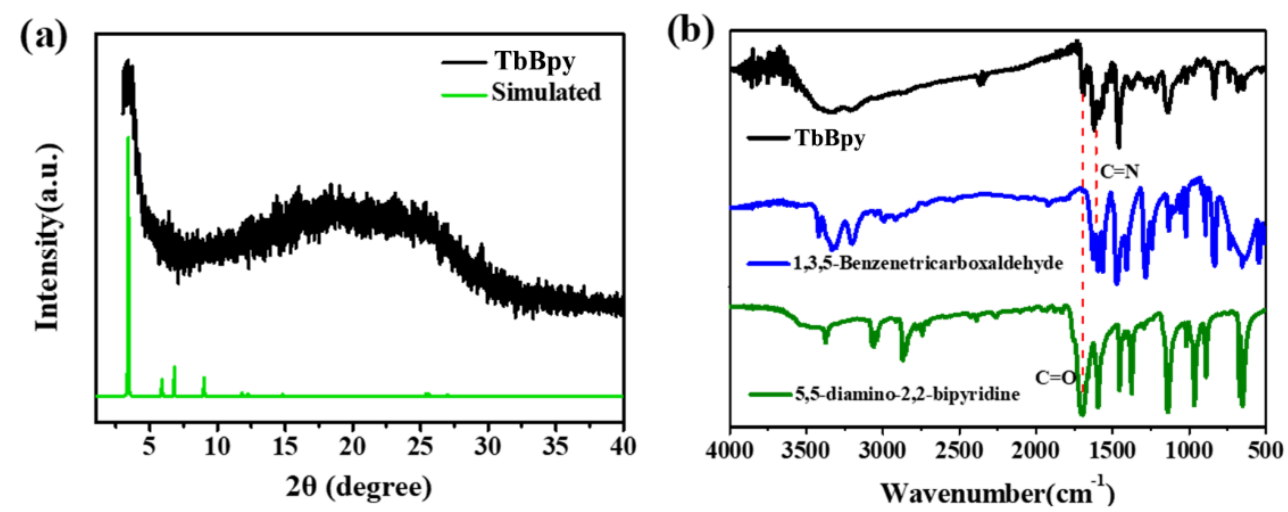

Figure S22. PXRD patterns (a) and FTIR spectra (b) of TbBpy.

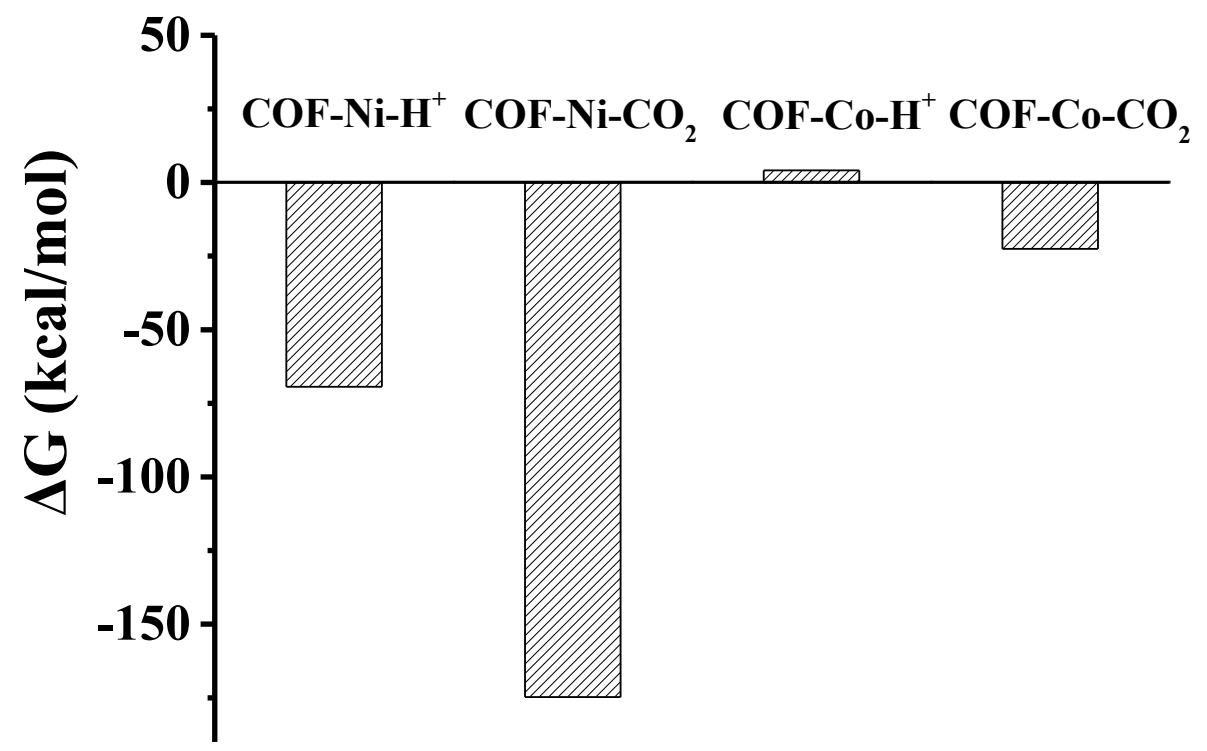

Figure S23. DFT-calculated relative Gibbs free energy $\left(\Delta G, \mathrm{kcal} \mathrm{mol}^{-1}\right)$ with Zero-point correction of $\mathrm{CO}_{2}$ and $\mathrm{H}^{+}$with the optimized building block of Ni-TpBpy and Co-TpBpy. 
Table S2. Photocatalytic $\mathrm{CO}_{2}$ reduction systems in aqueous solution for $\mathrm{CO}$ production.

\begin{tabular}{|c|c|c|c|c|c|c|}
\hline Photocatalyst & $\begin{array}{l}\text { CO }(\mu \text { mol } \\
\left.h^{-1} g^{-1}\right)\end{array}$ & TON & $\begin{array}{l}\text { Select. of } \\
\text { CO }\end{array}$ & Reaction solvent & $\begin{array}{l}\text { Irradiation } \\
\text { condition }\end{array}$ & Ref. \\
\hline $\begin{array}{l}\text { Ni-TpBpy } \\
{\left[\mathrm{Ru}(\mathrm{bpy})_{3}\right] \mathrm{Cl}_{2}}\end{array}$ & 966 & 13.6 & $96 \%$ & $\begin{array}{l}\mathrm{MeCN} / \mathrm{H}_{2} \mathrm{O} / \mathrm{TEOA} \\
(3: 1: 1)\end{array}$ & $\begin{array}{l}\lambda \geq 420 \mathrm{~nm} \\
(300 \mathrm{~W} \mathrm{Xe} \\
\text { lamp) }\end{array}$ & $\begin{array}{l}\text { This } \\
\text { work }\end{array}$ \\
\hline $\begin{array}{l}\text { Co-ZIF-9 } \\
{\left[\mathrm{Ru}(\mathrm{bpy})_{3}\right] \mathrm{Cl}_{2}}\end{array}$ & - & 89.6 & $58 \%$ & $\begin{array}{l}\mathrm{MeCN} / \mathrm{H}_{2} \mathrm{O} / \mathrm{TEOA} \\
(4: 1: 1)\end{array}$ & $\begin{array}{l}\lambda>420(\mathrm{Xe} \\
\text { lamp) }\end{array}$ & {$[4]$} \\
\hline $\begin{array}{l}\mathrm{Zr}-\mathrm{UiO}-66-\mathrm{NH}_{2} \\
{\left[\mathrm{Ru}(\mathrm{bpy})_{3}\right] \mathrm{Cl}_{2}}\end{array}$ & - & 4.3 & 35 & $\begin{array}{l}\mathrm{MeCN} / \mathrm{H}_{2} \mathrm{O} / \mathrm{TEOA} \\
(4: 1: 1)\end{array}$ & $\begin{array}{l}\lambda>420(\mathrm{Xe} \\
\text { lamp })\end{array}$ & {$[4]$} \\
\hline $\begin{array}{l}\text { Re3-MOF } \\
\text { Ag nanocubes }\end{array}$ & - & 2.8 & $100 \%$ & $\begin{array}{l}\mathrm{MeCN} / \mathrm{TEA} \\
(20: 1)\end{array}$ & $\begin{array}{l}\lambda=400-700 \\
\mathrm{~nm}(300 \mathrm{~W} \mathrm{Xe} \\
\text { lamp) }\end{array}$ & {$[5]$} \\
\hline $\begin{array}{l}\mathrm{Zr}_{6} \mathrm{O}_{4}(\mathrm{OH})_{4}[\mathrm{Re}(5, \\
\left.5^{\prime} \text {-bpydb }\right)(\mathrm{CO})_{3} \mathrm{Cl} \\
]_{6}\end{array}$ & - & 6.44 & $94 \%$ & $\begin{array}{l}\mathrm{MeCN} / \mathrm{TEA} / \mathrm{H}_{2} \mathrm{O} \\
(20: 1: 0.3)\end{array}$ & $410 \mathrm{~nm}$ LED & [6] \\
\hline $\begin{array}{l}\mathrm{Ni}_{3}(\mathrm{HITP})_{2} \\
\mathrm{Ru}(\mathrm{bpy})_{3} \mathrm{Cl}_{2}\end{array}$ & $3.45 \times 10^{4}$ & - & $97 \%$ & $\begin{array}{l}\mathrm{MeCN} / \mathrm{H}_{2} \mathrm{O} / \mathrm{TEOA} \\
(10: 2: 4)\end{array}$ & $\begin{array}{l}100 \mathrm{~W} \text { LED } \\
\text { light }(\lambda=420 \\
\mathrm{nm})\end{array}$ & [7] \\
\hline $\begin{array}{l}\text { spongy } \\
\mathrm{Ni}(\mathrm{TPA} / \mathrm{TEG}) \\
\text { nanostructure } \\
\mathrm{Ru}(\mathrm{bpy})_{3} \mathrm{Cl}_{2}\end{array}$ & $1.6 \times 10^{4}$ & - & nearly $100 \%$ & $\begin{array}{l}\mathrm{MeCN} / \mathrm{H}_{2} \mathrm{O} / \mathrm{TEOA} \\
(8: 2: 2)\end{array}$ & $\begin{array}{l}300 \mathrm{~W} \text { Xe } \\
\text { lamp }(\lambda>420 \\
\mathrm{nm})\end{array}$ & {$[8]$} \\
\hline Re-COF & $\begin{array}{l}\sim 15 \mathrm{mmol} \\
\mathrm{CO} / \mathrm{g} \\
>20 \mathrm{~h}\end{array}$ & 48 & $98 \%$ & $\begin{array}{l}\mathrm{MeCN} / \mathrm{TEOA} \\
(3: 0.2)\end{array}$ & $\begin{array}{l}225 \mathrm{~W} X \mathrm{Xe} \\
\text { lamp }(\lambda>420 \\
\mathrm{nm})\end{array}$ & [9] \\
\hline $\begin{array}{l}\text { MAF-X27-OH } \\
\mathrm{Ru}(\mathrm{bpy})_{3} \mathrm{Cl}_{2}\end{array}$ & - & $\begin{array}{l}28 \times 10 \\
-3 \mathrm{~s}^{-1} \\
(\mathrm{TOF})\end{array}$ & $97 \%$ & $\begin{array}{l}\mathrm{MeCN} / \mathrm{H}_{2} \mathrm{O} / \mathrm{TEOA}( \\
4: 1: 0.1)\end{array}$ & $\begin{array}{l}\text { LED light }(\lambda= \\
420 \mathrm{~nm})\end{array}$ & [10] \\
\hline $\begin{array}{l}\mathrm{Co}_{1}-\mathrm{G} \\
\mathrm{Ru}(\text { bpy })_{3} \mathrm{Cl}_{2}\end{array}$ & - & 374 & $79 \%$ & $\begin{array}{l}\mathrm{MeCN} / \mathrm{H}_{2} \mathrm{O} / \mathrm{TEOA}( \\
3: 1: 1)\end{array}$ & $\begin{array}{l}300 \mathrm{~W} \text { Xe } \\
\text { lamp }(\lambda>420 \\
\mathrm{nm})\end{array}$ & {$[11]$} \\
\hline $\begin{array}{l}\mathrm{Ni} \text { MOLs } \\
\mathrm{Ru}(\text { bpy })_{3} \mathrm{Cl}_{2}\end{array}$ & $12.5 \mu \mathrm{mol} / \mathrm{h}$ & - & $97.8 \%$ & $\begin{array}{l}\mathrm{MeCN} / \mathrm{H}_{2} \mathrm{O} / \mathrm{TEOA}( \\
3: 2: 1)\end{array}$ & $\begin{array}{l}5 \mathrm{~W} \text { white } \\
\text { LED light (400 } \\
\mathrm{nm} \leq \lambda \leq 800 \\
\mathrm{~nm})\end{array}$ & {$[12]$} \\
\hline $\begin{array}{l}\mathrm{Co}_{3} \mathrm{O}_{4} \text { hexagonal } \\
\text { platelets } \\
\mathrm{Ru}(\mathrm{bpy})_{3} \mathrm{Cl}_{2}\end{array}$ & 2003 & - & $77.1 \%$ & $\begin{array}{l}\mathrm{MeCN} / \mathrm{H}_{2} \mathrm{O} / \mathrm{TEOA}( \\
3: 1: 1)\end{array}$ & $\begin{array}{l}300 \mathrm{~W} X \mathrm{Xe} \\
\text { lamp }(\lambda>420 \\
\mathrm{nm})\end{array}$ & [13] \\
\hline $\begin{array}{l}\text { PCP-RuII } \\
\mathrm{Ru}(\text { bpy })_{3} \mathrm{Cl}_{2}\end{array}$ & - & 8.1 & $\begin{array}{l}15 \% \text { over } \\
\mathrm{HOOH} \text { and } \\
\mathrm{H}_{2}\end{array}$ & $\begin{array}{l}\mathrm{MeCN} / \mathrm{TEOA} \\
(14: 1)\end{array}$ & $\begin{array}{l}300 \mathrm{~W} \mathrm{Xe} \\
\text { lamp }(\lambda=385- \\
740 \mathrm{~nm})\end{array}$ & {$[14]$} \\
\hline
\end{tabular}


Calculated Geometries Parameters of Complexes by Gaussian 09, $\mathrm{L}$ is 2,2'-bipyridine.

Cartesian coordinates of $\mathrm{CO}_{2}$

$\begin{array}{lllr}\mathrm{C} & 0.000000 & 0.000000 & 0.000000 \\ \mathrm{O} & 0.000000 & 0.000000 & 1.159369 \\ \mathrm{O} & 0.000000 & 0.000000 & -1.159369\end{array}$

\section{Cartesian coordinates of $\mathrm{H}_{2} \mathrm{O}$}

$\begin{array}{llll}\mathrm{O} & 0.000000 & 0.000000 & 0.119640 \\ \mathrm{H} & 0.000000 & 0.754135 & -0.478562 \\ \mathrm{H} & 0.000000 & -0.754135 & -0.478562\end{array}$

\section{Cartesian coordinates of $\mathrm{CO}$}

$\begin{array}{llll}\mathrm{C} & 0.000000 & 0.000000 & -0.644610 \\ \mathrm{O} & 0.000000 & 0.000000 & 0.483458\end{array}$

\begin{tabular}{lrrr}
\multicolumn{4}{l}{ Cartesian coordinates of $\mathbf{L}_{\mathbf{2}} \mathbf{N i} \mathbf{}^{\mathbf{l}}$} \\
$\mathrm{C}$ & -2.366731 & 3.242054 & -1.210429 \\
$\mathrm{C}$ & -3.666619 & 2.760850 & -0.934799 \\
$\mathrm{C}$ & -3.807046 & 1.478566 & -0.448162 \\
$\mathrm{C}$ & -2.668834 & 0.677433 & -0.233109 \\
$\mathrm{~N}$ & -1.407015 & 1.166450 & -0.479731 \\
$\mathrm{C}$ & -1.288421 & 2.420518 & -0.965193 \\
$\mathrm{C}$ & -2.668849 & -0.677353 & 0.233066 \\
$\mathrm{C}$ & -3.807079 & -1.478465 & 0.448101 \\
$\mathrm{C}$ & -3.666683 & -2.760754 & 0.934735 \\
$\mathrm{C}$ & -2.366807 & -3.241985 & 1.210377 \\
$\mathrm{C}$ & -1.288479 & -2.420467 & 0.965160 \\
$\mathrm{~N}$ & -1.407043 & -1.166394 & 0.479704 \\
$\mathrm{H}$ & -2.205346 & 4.237330 & -1.627972 \\
$\mathrm{H}$ & -4.542425 & 3.390365 & -1.104767 \\
$\mathrm{H}$ & -4.796382 & 1.078409 & -0.219531 \\
$\mathrm{H}$ & -0.269101 & 2.750544 & -1.180581 \\
$\mathrm{H}$ & -4.796405 & -1.078288 & 0.219459 \\
$\mathrm{H}$ & -4.542503 & -3.390253 & 1.104688 \\
$\mathrm{H}$ & -2.205445 & -4.237266 & 1.627916 \\
$\mathrm{H}$ & -0.269168 & -2.750514 & 1.180559 \\
$\mathrm{C}$ & 2.366766 & 3.242014 & 1.210426 \\
$\mathrm{C}$ & 3.666647 & 2.760787 & 0.934799 \\
$\mathrm{C}$ & 3.807052 & 1.478500 & 0.448162 \\
$\mathrm{C}$ & 2.668827 & 0.677387 & 0.233107 \\
& & &
\end{tabular}




$\begin{array}{lrrr}\mathrm{N} & 1.407016 & 1.166425 & 0.479727 \\ \mathrm{C} & 1.288442 & 2.420496 & 0.965189 \\ \mathrm{C} & 2.668819 & -0.677399 & -0.233068 \\ \mathrm{C} & 3.807036 & -1.478532 & -0.448099 \\ \mathrm{C} & 3.666619 & -2.760818 & -0.934733 \\ \mathrm{C} & 2.366735 & -3.242025 & -1.210380 \\ \mathrm{C} & 1.288420 & -2.420489 & -0.965168 \\ \mathrm{~N} & 1.407005 & -1.166417 & -0.479712 \\ \mathrm{H} & 2.205397 & 4.237292 & 1.627968 \\ \mathrm{H} & 4.542463 & 3.390286 & 1.104769 \\ \mathrm{H} & 4.796382 & 1.078326 & 0.219533 \\ \mathrm{H} & 0.269128 & 2.750539 & 1.180575 \\ \mathrm{H} & 4.796367 & -1.078373 & -0.219453 \\ \mathrm{H} & 4.542427 & -3.390333 & -1.104682 \\ \mathrm{H} & 2.205356 & -4.237304 & -1.627918 \\ \mathrm{H} & 0.269104 & -2.750516 & -1.180571 \\ \mathrm{Ni} & -0.000009 & 0.000015 & -0.000003\end{array}$

Cartesian coordinates of $\mathrm{L}_{2} \mathrm{Ni}-\mathrm{CO}_{2}$

$\begin{array}{lrrr}\mathrm{C} & -2.446693 & -3.202568 & 1.558543 \\ \mathrm{C} & -3.725406 & -2.714026 & 1.211366 \\ \mathrm{C} & -3.830624 & -1.431584 & 0.715479 \\ \mathrm{C} & -2.677461 & -0.638454 & 0.565258 \\ \mathrm{~N} & -1.436648 & -1.130869 & 0.888790 \\ \mathrm{C} & -1.351308 & -2.387108 & 1.375669 \\ \mathrm{C} & -2.645058 & 0.716392 & 0.095366 \\ \mathrm{C} & -3.766719 & 1.525066 & -0.167184 \\ \mathrm{C} & -3.596419 & 2.809231 & -0.640588 \\ \mathrm{C} & -2.282897 & 3.281923 & -0.855747 \\ \mathrm{C} & -1.221350 & 2.452766 & -0.567633 \\ \mathrm{~N} & -1.370656 & 1.196801 & -0.092309 \\ \mathrm{H} & -2.314923 & -4.199862 & 1.981677 \\ \mathrm{H} & -4.612796 & -3.338811 & 1.331051 \\ \mathrm{H} & -4.802273 & -1.027401 & 0.426664 \\ \mathrm{H} & -0.347161 & -2.722722 & 1.646474 \\ \mathrm{H} & -4.767763 & 1.130238 & 0.014554 \\ \mathrm{H} & -4.459653 & 3.445405 & -0.846100 \\ \mathrm{H} & -2.096086 & 4.277589 & -1.261574 \\ \mathrm{H} & -0.192001 & 2.771607 & -0.747686 \\ \mathrm{C} & 2.279118 & -3.286946 & -0.849844 \\ \mathrm{C} & 3.594512 & -2.812553 & -0.650450 \\ \mathrm{C} & 3.769030 & -1.524569 & -0.189115 \\ \mathrm{C} & 2.649754 & -0.713753 & 0.076996 \\ \mathrm{~N} & 1.373726 & -1.195652 & -0.095159\end{array}$




$\begin{array}{lrrr}\mathrm{C} & 1.220187 & -2.455441 & -0.558877 \\ \mathrm{C} & 2.686357 & 0.644870 & 0.535542 \\ \mathrm{C} & 3.840786 & 1.439197 & 0.668768 \\ \mathrm{C} & 3.740006 & 2.725633 & 1.155146 \\ \mathrm{C} & 2.464479 & 3.216981 & 1.510005 \\ \mathrm{C} & 1.367530 & 2.400057 & 1.343793 \\ \mathrm{~N} & 1.448512 & 1.139903 & 0.866378 \\ \mathrm{H} & 2.088684 & -4.285874 & -1.245854 \\ \mathrm{H} & 4.455880 & -3.450402 & -0.858585 \\ \mathrm{H} & 4.771660 & -1.128293 & -0.019692 \\ \mathrm{H} & 0.189266 & -2.775654 & -0.727087 \\ \mathrm{H} & 4.809784 & 1.032682 & 0.374375 \\ \mathrm{H} & 4.628403 & 3.351378 & 1.261615 \\ \mathrm{H} & 2.336503 & 4.217688 & 1.926190 \\ \mathrm{H} & 0.365869 & 2.737868 & 1.621005 \\ \mathrm{Ni} & 0.003932 & 0.002690 & 0.433984 \\ \mathrm{C} & -0.012490 & -0.010667 & -2.634461 \\ \mathrm{O} & -0.785882 & -0.875935 & -2.665869 \\ \mathrm{O} & 0.760481 & 0.854238 & -2.681906\end{array}$

\section{Cartesian coordinates of $\mathrm{L}_{2} \mathrm{Ni}-\mathrm{H}_{2} \mathrm{O}$}

$\begin{array}{lrrr}\mathrm{C} & -2.615934 & 3.159901 & -1.565290 \\ \mathrm{C} & -3.889286 & 2.589435 & -1.361915 \\ \mathrm{C} & -3.966946 & 1.316238 & -0.833245 \\ \mathrm{C} & -2.790396 & 0.618293 & -0.509916 \\ \mathrm{~N} & -1.557251 & 1.189302 & -0.697551 \\ \mathrm{C} & -1.497144 & 2.431964 & -1.218536 \\ \mathrm{C} & -2.723817 & -0.715623 & 0.028762 \\ \mathrm{C} & -3.811344 & -1.587229 & 0.212008 \\ \mathrm{C} & -3.606038 & -2.827543 & 0.784137 \\ \mathrm{C} & -2.302389 & -3.181738 & 1.185602 \\ \mathrm{C} & -1.269308 & -2.293563 & 0.967138 \\ \mathrm{~N} & -1.455203 & -1.090797 & 0.383167 \\ \mathrm{H} & -2.501410 & 4.152801 & -2.003551 \\ \mathrm{H} & -4.795884 & 3.142314 & -1.615905 \\ \mathrm{H} & -4.936575 & 0.848444 & -0.654711 \\ \mathrm{H} & -0.492439 & 2.833002 & -1.374161 \\ \mathrm{H} & -4.809858 & -1.280134 & -0.103662 \\ \mathrm{H} & -4.441562 & -3.514943 & 0.931961 \\ \mathrm{H} & -2.099770 & -4.130547 & 1.685251 \\ \mathrm{H} & -0.251544 & -2.494393 & 1.314420 \\ \mathrm{C} & 2.099301 & 3.510584 & 1.015281 \\ \mathrm{C} & 3.423547 & 3.085914 & 0.757956 \\ \mathrm{C} & 3.629511 & 1.796483 & 0.317181\end{array}$




$\begin{array}{lrrr}\mathrm{C} & 2.534192 & 0.931083 & 0.129088 \\ \mathrm{~N} & 1.247373 & 1.360623 & 0.367824 \\ \mathrm{C} & 1.065019 & 2.627762 & 0.804683 \\ \mathrm{C} & 2.601806 & -0.434975 & -0.292989 \\ \mathrm{C} & 3.776078 & -1.204737 & -0.421628 \\ \mathrm{C} & 3.697484 & -2.519296 & -0.829134 \\ \mathrm{C} & 2.424447 & -3.065889 & -1.112187 \\ \mathrm{C} & 1.310184 & -2.272628 & -0.956068 \\ \mathrm{~N} & 1.366815 & -0.983046 & -0.558327 \\ \mathrm{H} & 1.887833 & 4.511733 & 1.394749 \\ \mathrm{H} & 4.265582 & 3.765302 & 0.904110 \\ \mathrm{H} & 4.637848 & 1.440597 & 0.097942 \\ \mathrm{H} & 0.029596 & 2.910954 & 1.010514 \\ \mathrm{H} & 4.741195 & -0.755661 & -0.180018 \\ \mathrm{H} & 4.599861 & -3.126012 & -0.927649 \\ \mathrm{H} & 2.312873 & -4.093817 & -1.460997 \\ \mathrm{H} & 0.310372 & -2.657725 & -1.170970 \\ \mathrm{Ni} & -0.100644 & 0.128433 & -0.116137 \\ \mathrm{O} & 1.404829 & -1.269691 & 2.535866 \\ \mathrm{H} & 2.227639 & -1.377760 & 2.045765 \\ \mathrm{H} & 0.960800 & -0.567133 & 2.036694\end{array}$

\begin{tabular}{lrrr}
\multicolumn{4}{c}{ Cartesian coordinates of $\mathbf{L 2 N i} \mathbf{2}^{\mathbf{}}$} \\
$\mathrm{C}$ & -2.348606 & 3.136085 & -1.468767 \\
$\mathrm{C}$ & -3.631447 & 2.618164 & -1.309028 \\
$\mathrm{C}$ & -3.788418 & 1.365386 & -0.719541 \\
$\mathrm{C}$ & -2.660190 & 0.675235 & -0.286872 \\
$\mathrm{~N}$ & -1.421221 & 1.210887 & -0.401610 \\
$\mathrm{C}$ & -1.266977 & 2.396570 & -1.004827 \\
$\mathrm{C}$ & -2.660207 & -0.675157 & 0.286824 \\
$\mathrm{C}$ & -3.788454 & -1.365287 & 0.719474 \\
$\mathrm{C}$ & -3.631516 & -2.618069 & 1.308963 \\
$\mathrm{C}$ & -2.348688 & -3.136014 & 1.468721 \\
$\mathrm{C}$ & -1.267038 & -2.396519 & 1.004799 \\
$\mathrm{~N}$ & -1.421250 & -1.210832 & 0.401581 \\
$\mathrm{H}$ & -2.178242 & 4.099754 & -1.952201 \\
$\mathrm{H}$ & -4.504187 & 3.174782 & -1.658651 \\
$\mathrm{H}$ & -4.779979 & 0.921879 & -0.620802 \\
$\mathrm{H}$ & -0.245275 & 2.759706 & -1.128791 \\
$\mathrm{H}$ & -4.780006 & -0.921762 & 0.620721 \\
$\mathrm{H}$ & -4.504273 & -3.174671 & 1.658572 \\
$\mathrm{H}$ & -2.178350 & -4.099687 & 1.952157 \\
$\mathrm{H}$ & -0.245344 & -2.759675 & 1.128778 \\
$\mathrm{C}$ & 2.348641 & 3.136045 & 1.468764
\end{tabular}




$\begin{array}{lrrr}\mathrm{C} & 3.631472 & 2.618102 & 1.309027 \\ \mathrm{C} & 3.788422 & 1.365320 & 0.719540 \\ \mathrm{C} & 2.660183 & 0.675189 & 0.286870 \\ \mathrm{~N} & 1.421223 & 1.210862 & 0.401607 \\ \mathrm{C} & 1.266999 & 2.396549 & 1.004824 \\ \mathrm{C} & 2.660177 & -0.675203 & -0.286825 \\ \mathrm{C} & 3.788413 & -1.365353 & -0.719474 \\ \mathrm{C} & 3.631454 & -2.618132 & -1.308963 \\ \mathrm{C} & 2.348617 & -3.136054 & -1.468724 \\ \mathrm{C} & 1.266978 & -2.396540 & -1.004804 \\ \mathrm{~N} & 1.421210 & -1.210856 & -0.401585 \\ \mathrm{H} & 2.178293 & 4.099717 & 1.952198 \\ \mathrm{H} & 4.504222 & 3.174705 & 1.658651 \\ \mathrm{H} & 4.779976 & 0.921797 & 0.620803 \\ \mathrm{H} & 0.245303 & 2.759702 & 1.128787 \\ \mathrm{H} & 4.779972 & -0.921845 & -0.620718 \\ \mathrm{H} & 4.504201 & -3.174749 & -1.658571 \\ \mathrm{H} & 2.178262 & -4.099724 & -1.952160 \\ \mathrm{H} & 0.245279 & -2.759677 & -1.128785 \\ \mathrm{Ni} & -0.000009 & 0.000015 & -0.000002\end{array}$

\begin{tabular}{lrrr}
\multicolumn{4}{l}{ Cartesian coordinates of $\mathbf{L}_{2} \mathbf{N i}-\mathbf{C O}^{2+}$} \\
$\mathrm{C}$ & -2.922545 & 3.215679 & -0.846957 \\
$\mathrm{C}$ & -4.130014 & 2.526091 & -0.770099 \\
$\mathrm{C}$ & -4.117843 & 1.176297 & -0.424198 \\
$\mathrm{C}$ & -2.901096 & 0.561403 & -0.145672 \\
$\mathrm{~N}$ & -1.733610 & 1.247834 & -0.194442 \\
$\mathrm{C}$ & -1.743389 & 2.535574 & -0.562405 \\
$\mathrm{C}$ & -2.725984 & -0.856470 & 0.186097 \\
$\mathrm{C}$ & -3.734659 & -1.729085 & 0.584247 \\
$\mathrm{C}$ & -3.392839 & -3.029046 & 0.954521 \\
$\mathrm{C}$ & -2.054305 & -3.414913 & 0.935492 \\
$\mathrm{C}$ & -1.102186 & -2.493417 & 0.515188 \\
$\mathrm{~N}$ & -1.437002 & -1.259293 & 0.122887 \\
$\mathrm{H}$ & -2.885066 & 4.269162 & -1.133014 \\
$\mathrm{H}$ & -5.073768 & 3.028135 & -0.999934 \\
$\mathrm{H}$ & -5.045217 & 0.601202 & -0.394973 \\
$\mathrm{H}$ & -0.777294 & 3.035439 & -0.649755 \\
$\mathrm{H}$ & -4.771779 & -1.392122 & 0.634366 \\
$\mathrm{H}$ & -4.166133 & -3.725349 & 1.290422 \\
$\mathrm{H}$ & -1.743385 & -4.410589 & 1.258988 \\
$\mathrm{H}$ & -0.034628 & -2.716056 & 0.513104 \\
$\mathrm{C}$ & 2.091510 & 3.155930 & 1.624097 \\
$\mathrm{C}$ & 3.372057 & 2.838291 & 1.175770
\end{tabular}




$\begin{array}{lrrr}\mathrm{C} & 3.542018 & 1.768215 & 0.299561 \\ \mathrm{C} & 2.420386 & 1.056808 & -0.121310 \\ \mathrm{~N} & 1.178086 & 1.402562 & 0.288050 \\ \mathrm{C} & 1.016548 & 2.406442 & 1.160646 \\ \mathrm{C} & 2.441023 & -0.138229 & -0.971872 \\ \mathrm{C} & 3.531845 & -0.565679 & -1.723183 \\ \mathrm{C} & 3.397869 & -1.698004 & -2.523994 \\ \mathrm{C} & 2.173519 & -2.359370 & -2.561883 \\ \mathrm{C} & 1.124816 & -1.872191 & -1.788513 \\ \mathrm{~N} & 1.261881 & -0.803470 & -0.995248 \\ \mathrm{H} & 1.922653 & 3.969992 & 2.332448 \\ \mathrm{H} & 4.239401 & 3.404701 & 1.525953 \\ \mathrm{H} & 4.540164 & 1.475775 & -0.031838 \\ \mathrm{H} & 0.000697 & 2.607449 & 1.507140 \\ \mathrm{H} & 4.469318 & -0.006599 & -1.705617 \\ \mathrm{H} & 4.238061 & -2.047233 & -3.130345 \\ \mathrm{H} & 2.021172 & -3.239756 & -3.190200 \\ \mathrm{H} & 0.141137 & -2.344263 & -1.821094 \\ \mathrm{Ni} & -0.173824 & 0.134077 & -0.132792 \\ \mathrm{C} & 2.078169 & -2.050056 & 2.612012 \\ \mathrm{O} & 2.839609 & -2.072886 & 3.439819\end{array}$

\section{Cartesian coordinates of $\mathrm{L}_{2} \mathrm{NiCO}_{2} \mathrm{H}^{+}$}

$\begin{array}{lrrr}\text { C } & -1.612834 & 3.244888 & -2.032794 \\ \mathrm{C} & -2.947443 & 2.848616 & -2.024394 \\ \mathrm{C} & -3.291806 & 1.638225 & -1.429282 \\ \mathrm{C} & -2.279079 & 0.858998 & -0.861563 \\ \mathrm{~N} & -0.999099 & 1.246539 & -0.880128 \\ \mathrm{C} & -0.672634 & 2.404168 & -1.439883 \\ \mathrm{C} & -2.539552 & -0.426661 & -0.171600 \\ \mathrm{C} & -3.746440 & -1.123963 & -0.279204 \\ \mathrm{C} & -3.917445 & -2.314261 & 0.417912 \\ \mathrm{C} & -2.879692 & -2.784420 & 1.219623 \\ \mathrm{C} & -1.706688 & -2.044638 & 1.283336 \\ \mathrm{~N} & -1.539940 & -0.909667 & 0.594788 \\ \mathrm{H} & -1.303251 & 4.189289 & -2.483992 \\ \mathrm{H} & -3.719246 & 3.481293 & -2.468206 \\ \mathrm{H} & -4.335985 & 1.326273 & -1.389921 \\ \mathrm{H} & 0.388208 & 2.674423 & -1.414903 \\ \mathrm{H} & -4.543969 & -0.741675 & -0.916225 \\ \mathrm{H} & -4.855344 & -2.868247 & 0.337612 \\ \mathrm{H} & -2.973567 & -3.706434 & 1.795262 \\ \mathrm{H} & -0.872926 & -2.349556 & 1.917466 \\ \mathrm{C} & 3.542133 & 2.406841 & 1.252815\end{array}$




$\begin{array}{lrrc}\mathrm{C} & 4.530454 & 1.698045 & 0.578115 \\ \mathrm{C} & 4.177987 & 0.525486 & -0.081749 \\ \mathrm{C} & 2.857381 & 0.081817 & -0.029065 \\ \mathrm{~N} & 1.914374 & 0.752362 & 0.674995 \\ \mathrm{C} & 2.246296 & 1.902240 & 1.276408 \\ \mathrm{C} & 2.355268 & -1.084235 & -0.772246 \\ \mathrm{C} & 3.152002 & -1.936770 & -1.538951 \\ \mathrm{C} & 2.548633 & -2.967632 & -2.250590 \\ \mathrm{C} & 1.164754 & -3.113104 & -2.184952 \\ \mathrm{C} & 0.441851 & -2.231206 & -1.390524 \\ \mathrm{~N} & 1.020182 & -1.250642 & -0.694020 \\ \mathrm{H} & 3.760928 & 3.344260 & 1.766601 \\ \mathrm{H} & 5.560805 & 2.058885 & 0.549910 \\ \mathrm{H} & 4.923978 & -0.031415 & -0.648664 \\ \mathrm{H} & 1.431035 & 2.420772 & 1.787410 \\ \mathrm{H} & 4.232590 & -1.799597 & -1.581091 \\ \mathrm{H} & 3.153533 & -3.647343 & -2.854533 \\ \mathrm{H} & 0.647342 & -3.898738 & -2.737806 \\ \mathrm{H} & -0.643962 & -2.312181 & -1.307411 \\ \mathrm{Ni} & 0.158038 & -0.017480 & 0.612999 \\ \mathrm{C} & -0.419891 & 0.846970 & 2.180765 \\ \mathrm{O} & -0.517334 & 2.041127 & 2.345355 \\ \mathrm{O} & -0.740127 & -0.007765 & 3.171566 \\ \mathrm{H} & -1.021139 & 0.530665 & 3.933734\end{array}$

\section{Cartesian coordinates of $\mathrm{L}_{2} \mathrm{NiH}^{+}$}

$\begin{array}{lrrr}\mathrm{C} & -2.230990 & 2.892031 & -1.464763 \\ \mathrm{C} & -3.491937 & 2.315590 & -1.332307 \\ \mathrm{C} & -3.638859 & 1.181607 & -0.539083 \\ \mathrm{C} & -2.510348 & 0.657146 & 0.099885 \\ \mathrm{~N} & -1.304481 & 1.222833 & -0.028963 \\ \mathrm{C} & -1.165552 & 2.305856 & -0.784466 \\ \mathrm{C} & -2.552729 & -0.543961 & 0.967129 \\ \mathrm{C} & -3.656254 & -1.399316 & 1.040900 \\ \mathrm{C} & -3.611871 & -2.508113 & 1.877330 \\ \mathrm{C} & -2.461029 & -2.742556 & 2.626559 \\ \mathrm{C} & -1.401953 & -1.852601 & 2.506604 \\ \mathrm{~N} & -1.446433 & -0.787426 & 1.698039 \\ \mathrm{H} & -2.073388 & 3.780507 & -2.078957 \\ \mathrm{H} & -4.358222 & 2.747824 & -1.838002 \\ \mathrm{H} & -4.622831 & 0.729398 & -0.411142 \\ \mathrm{H} & -0.156252 & 2.725669 & -0.851300 \\ \mathrm{H} & -4.542052 & -1.205970 & 0.435945 \\ \mathrm{H} & -4.467760 & -3.183414 & 1.942218\end{array}$




$\begin{array}{lrrr}\mathrm{H} & -2.381034 & -3.596862 & 3.300509 \\ \mathrm{H} & -0.484533 & -1.982009 & 3.084400 \\ \mathrm{C} & 2.982840 & 3.152976 & 2.668908 \\ \mathrm{C} & 4.071322 & 2.736054 & 1.906321 \\ \mathrm{C} & 3.923806 & 1.629151 & 1.077928 \\ \mathrm{C} & 2.697326 & 0.966503 & 1.035843 \\ \mathrm{~N} & 1.646957 & 1.373185 & 1.790395 \\ \mathrm{C} & 1.790519 & 2.445381 & 2.583254 \\ \mathrm{C} & 2.411536 & -0.204129 & 0.192844 \\ \mathrm{C} & 3.322241 & -0.800984 & -0.681162 \\ \mathrm{C} & 2.916673 & -1.899574 & -1.430889 \\ \mathrm{C} & 1.611029 & -2.368398 & -1.294453 \\ \mathrm{C} & 0.762624 & -1.719647 & -0.405555 \\ \mathrm{~N} & 1.153465 & -0.673039 & 0.323959 \\ \mathrm{H} & 3.050020 & 4.016146 & 3.333051 \\ \mathrm{H} & 5.024958 & 3.265714 & 1.955308 \\ \mathrm{H} & 4.757967 & 1.282948 & 0.467299 \\ \mathrm{H} & 0.913604 & 2.726033 & 3.165995 \\ \mathrm{H} & 4.336736 & -0.413482 & -0.777356 \\ \mathrm{H} & 3.613484 & -2.383948 & -2.118260 \\ \mathrm{H} & 1.250822 & -3.224161 & -1.867638 \\ \mathrm{H} & -0.270716 & -2.046757 & -0.266520 \\ \mathrm{Ni} & 0.064799 & 0.384335 & 1.579575 \\ \mathrm{H} & -0.565145 & 1.100378 & 2.676130\end{array}$

\section{Cartesian coordinates of COF model.}

$\begin{array}{llcc}\mathrm{O} & -7.005132 & -1.646416 & 2.006187 \\ \mathrm{C} & -7.005087 & -1.687721 & -0.345928 \\ \mathrm{C} & -7.067786 & 3.922077 & -1.361778 \\ \mathrm{C} & -7.004965 & 2.890568 & 1.184610 \\ \mathrm{C} & -7.036487 & 2.003013 & 0.104811 \\ \mathrm{H} & -6.972968 & 2.472732 & 2.195425 \\ \mathrm{C} & -7.087113 & 2.542218 & -1.189240 \\ \mathrm{~N} & -7.004991 & 0.622669 & 0.357840 \\ \mathrm{H} & -7.131374 & 1.903303 & -2.071719 \\ \mathrm{H} & -7.093832 & 4.329095 & -2.375372 \\ \mathrm{C} & -7.005052 & -0.322689 & -0.567882 \\ \mathrm{H} & -6.992948 & 0.253770 & 1.320412 \\ \mathrm{H} & -6.999497 & 0.011776 & -1.608957 \\ \mathrm{C} & -3.508999 & -2.320418 & 0.974341 \\ \mathrm{O} & -3.502586 & -1.646606 & 2.006363 \\ \mathrm{C} & -3.502598 & -1.687806 & -0.345894 \\ \mathrm{C} & -3.517647 & 3.921509 & -1.361690 \\ \mathrm{C} & -3.502434 & 2.890462 & 1.184584\end{array}$




\begin{tabular}{|c|c|c|c|}
\hline C & -3.509582 & 2.002351 & 0.105084 \\
\hline $\mathrm{H}$ & -3.494935 & 2.472392 & 2.195728 \\
\hline $\mathrm{C}$ & -3.521770 & 2.542182 & -1.189177 \\
\hline $\mathrm{N}$ & -3.502531 & 0.622570 & 0.357899 \\
\hline $\mathrm{H}$ & -3.532619 & 1.902807 & -2.072374 \\
\hline $\mathrm{H}$ & -3.525275 & 4.327868 & -2.375809 \\
\hline $\mathrm{C}$ & -3.502496 & -0.322823 & -0.567759 \\
\hline $\mathrm{H}$ & -3.498980 & 0.251169 & 1.319904 \\
\hline $\mathrm{H}$ & -3.500228 & 0.011241 & -1.609025 \\
\hline $\mathrm{C}$ & -0.000458 & -2.320655 & 0.974408 \\
\hline $\mathrm{O}$ & -0.000156 & -1.646731 & 2.006326 \\
\hline $\mathrm{C}$ & -0.000039 & -1.687970 & -0.345827 \\
\hline $\mathrm{C}$ & 0.000757 & 3.921278 & -1.361557 \\
\hline $\mathrm{C}$ & 0.000031 & 2.890330 & 1.184687 \\
\hline $\mathrm{C}$ & 0.000327 & 2.002085 & 0.105191 \\
\hline $\mathrm{H}$ & -0.000320 & 2.472192 & 2.195811 \\
\hline $\mathrm{C}$ & 0.000886 & 2.542014 & -1.189058 \\
\hline $\mathrm{N}$ & 0.000010 & 0.622415 & 0.357877 \\
\hline $\mathrm{H}$ & 0.001397 & 1.902480 & -2.072202 \\
\hline $\mathrm{H}$ & 0.001184 & 4.327606 & -2.375736 \\
\hline $\mathrm{C}$ & -0.000046 & -0.322999 & -0.567763 \\
\hline $\mathrm{H}$ & 0.000025 & 0.250766 & 1.319840 \\
\hline $\mathrm{H}$ & -0.000010 & 0.010942 & -1.609058 \\
\hline $\mathrm{C}$ & 3.509119 & -2.320590 & 0.974376 \\
\hline $\mathrm{O}$ & 3.502318 & -1.646732 & 2.006382 \\
\hline $\mathrm{C}$ & 3.502409 & -1.688011 & -0.345860 \\
\hline $\mathrm{C}$ & 3.519744 & 3.921265 & -1.361614 \\
\hline $\mathrm{C}$ & 3.502456 & 2.890216 & 1.184658 \\
\hline $\mathrm{C}$ & 3.510436 & 2.002106 & 0.105169 \\
\hline $\mathrm{H}$ & 3.493885 & 2.472162 & 2.195799 \\
\hline $\mathrm{C}$ & 3.524190 & 2.541940 & -1.189077 \\
\hline $\mathrm{N}$ & 3.502473 & 0.622320 & 0.357970 \\
\hline $\mathrm{H}$ & 3.536571 & 1.902556 & -2.072248 \\
\hline $\mathrm{H}$ & 3.528760 & 4.327614 & -2.375726 \\
\hline $\mathrm{C}$ & 3.502389 & -0.323035 & -0.567728 \\
\hline $\mathrm{H}$ & 3.498807 & 0.250868 & 1.319963 \\
\hline $\mathrm{H}$ & 3.499966 & 0.011054 & -1.608985 \\
\hline $\mathrm{C}$ & 7.025740 & -2.320653 & 0.973477 \\
\hline $\mathrm{O}$ & 7.004931 & -1.646854 & 2.006326 \\
\hline $\mathrm{C}$ & 7.004958 & -1.688124 & -0.345806 \\
\hline $\mathrm{C}$ & 7.068255 & 3.921656 & -1.361595 \\
\hline $\mathrm{C}$ & 7.005112 & 2.890103 & 1.184703 \\
\hline $\mathrm{C}$ & 7.036760 & 2.002548 & 0.104935 \\
\hline $\mathrm{H}$ & 6.972985 & 2.472265 & 2.195517 \\
\hline
\end{tabular}




\begin{tabular}{|c|c|c|c|}
\hline $\mathrm{C}$ & 7.087589 & 2.541787 & -1.189093 \\
\hline $\mathrm{N}$ & 7.005037 & 0.622212 & 0.358035 \\
\hline $\mathrm{H}$ & 7.132027 & 1.902902 & -2.071588 \\
\hline $\mathrm{H}$ & 7.094345 & 4.328681 & -2.375187 \\
\hline $\mathrm{C}$ & 7.005046 & -0.323131 & -0.567689 \\
\hline $\mathrm{H}$ & 6.993089 & 0.253452 & 1.320629 \\
\hline $\mathrm{H}$ & 6.999639 & 0.011406 & -1.608740 \\
\hline $\mathrm{C}$ & -0.000127 & -4.063216 & -1.426717 \\
\hline $\mathrm{H}$ & -0.000052 & -4.682260 & -2.322134 \\
\hline $\mathrm{C}$ & -0.000470 & -4.592257 & -0.089504 \\
\hline $\mathrm{H}$ & -0.001037 & -5.677165 & 0.058519 \\
\hline $\mathrm{C}$ & -0.000956 & -3.784663 & 1.010985 \\
\hline $\mathrm{H}$ & -0.001462 & -4.208070 & 2.017323 \\
\hline $\mathrm{C}$ & 0.000121 & -2.660387 & -1.493804 \\
\hline $\mathrm{H}$ & 0.000537 & -2.201257 & -2.491522 \\
\hline $\mathrm{C}$ & 3.502623 & 4.784985 & -0.268559 \\
\hline $\mathrm{H}$ & 3.496571 & 5.865489 & -0.427671 \\
\hline $\mathrm{C}$ & 7.005191 & 4.784859 & -0.268485 \\
\hline $\mathrm{H}$ & 6.978796 & 5.865023 & -0.428367 \\
\hline $\mathrm{C}$ & 0.000097 & 4.785089 & -0.268505 \\
\hline $\mathrm{H}$ & -0.000090 & 5.865615 & -0.427583 \\
\hline $\mathrm{C}$ & -3.502370 & 4.785221 & -0.268607 \\
\hline $\mathrm{H}$ & -3.496751 & 5.865730 & -0.427710 \\
\hline $\mathrm{C}$ & -7.004927 & 4.785277 & -0.268667 \\
\hline $\mathrm{H}$ & -6.978607 & 5.865443 & -0.428559 \\
\hline $\mathrm{C}$ & -0.000169 & 4.261603 & 1.013390 \\
\hline $\mathrm{H}$ & -0.000581 & 4.916026 & 1.887409 \\
\hline $\mathrm{C}$ & -3.497106 & 4.261788 & 1.013312 \\
\hline $\mathrm{H}$ & -3.487255 & 4.916217 & 1.887270 \\
\hline $\mathrm{C}$ & -6.988731 & 4.262467 & 1.013830 \\
\hline $\mathrm{H}$ & -6.948766 & 4.916575 & 1.887135 \\
\hline $\mathrm{C}$ & 3.496579 & 4.261545 & 1.013358 \\
\hline $\mathrm{H}$ & 3.485585 & 4.915972 & 1.887304 \\
\hline $\mathrm{C}$ & 6.988874 & 4.262007 & 1.013990 \\
\hline $\mathrm{H}$ & 6.948773 & 4.916072 & 1.887320 \\
\hline $\mathrm{C}$ & 3.519971 & -4.592240 & -0.089726 \\
\hline $\mathrm{H}$ & 3.528354 & -5.677138 & 0.058184 \\
\hline $\mathrm{C}$ & 3.502306 & -4.063171 & -1.426792 \\
\hline $\mathrm{H}$ & 3.494400 & -4.682222 & -2.322179 \\
\hline $\mathrm{C}$ & 3.496400 & -2.660292 & -1.493854 \\
\hline $\mathrm{H}$ & 3.483764 & -2.201231 & -2.491512 \\
\hline $\mathrm{C}$ & 3.523988 & -3.784591 & 1.010877 \\
\hline $\mathrm{H}$ & 3.534188 & -4.208098 & 2.017146 \\
\hline $\mathrm{C}$ & 7.058955 & -4.592630 & -0.091162 \\
\hline
\end{tabular}




$\begin{array}{llll}\mathrm{H} & 7.078184 & -5.677530 & 0.056101 \\ \mathrm{C} & 7.073678 & -3.783878 & 1.010196 \\ \mathrm{H} & 7.096444 & -4.208681 & 2.016080 \\ \mathrm{C} & 7.004863 & -4.063251 & -1.426669 \\ \mathrm{H} & 6.975877 & -4.682296 & -2.321788 \\ \mathrm{C} & 6.989981 & -2.658971 & -1.493583 \\ \mathrm{H} & 6.948870 & -2.200627 & -2.490651 \\ \mathrm{C} & -3.502696 & -4.063054 & -1.426783 \\ \mathrm{H} & -3.494939 & -4.682103 & -2.322172 \\ \mathrm{C} & -3.520276 & -4.592111 & -0.089712 \\ \mathrm{H} & -3.528625 & -5.677007 & 0.058218 \\ \mathrm{C} & -3.523757 & -3.784433 & 1.010861 \\ \mathrm{H} & -3.533699 & -4.207896 & 2.017151 \\ \mathrm{C} & -3.496822 & -2.660159 & -1.493866 \\ \mathrm{H} & -3.484404 & -2.201131 & -2.491543 \\ \mathrm{C} & -7.026612 & -2.320228 & 0.973336 \\ \mathrm{C} & -7.075778 & -3.783380 & 1.010066 \\ \mathrm{H} & -7.099362 & -4.208151 & 2.015948 \\ \mathrm{C} & -7.005140 & -4.062726 & -1.426746 \\ \mathrm{H} & -6.975688 & -4.681860 & -2.321795 \\ \mathrm{C} & -7.060820 & -4.592124 & -0.091322 \\ \mathrm{H} & -7.080833 & -5.677021 & 0.055845 \\ \mathrm{C} & -6.989703 & -2.658491 & -1.493713 \\ \mathrm{H} & -6.947722 & -2.200145 & -2.490738\end{array}$

\section{Cartesian coordinates of COF- $\mathrm{L}_{\mathbf{2}} \mathrm{Ni}$ complex with $\mathrm{COF}$ data omitted}

$\begin{array}{lrrl}\mathrm{C} & -0.374794 & 1.303557 & 4.726609 \\ \mathrm{C} & 0.962580 & 1.695799 & 4.558862 \\ \mathrm{C} & 1.833457 & 0.837706 & 3.919718 \\ \mathrm{C} & 1.362961 & -0.401849 & 3.458414 \\ \mathrm{~N} & 0.053937 & -0.760901 & 3.588901 \\ \mathrm{C} & -0.779656 & 0.085455 & 4.220158 \\ \mathrm{C} & 2.183806 & -1.438677 & 2.883580 \\ \mathrm{C} & 3.586786 & -1.423410 & 2.840832 \\ \mathrm{C} & 4.276273 & -2.541987 & 2.405919 \\ \mathrm{C} & 3.532489 & -3.656697 & 1.963498 \\ \mathrm{C} & 2.155777 & -3.583077 & 1.957354 \\ \mathrm{~N} & 1.464540 & -2.520229 & 2.429197 \\ \mathrm{H} & -1.098139 & 1.937272 & 5.242163 \\ \mathrm{H} & 1.309339 & 2.667782 & 4.916964 \\ \mathrm{H} & 2.864754 & 1.124720 & 3.718119 \\ \mathrm{H} & -1.811351 & -0.251220 & 4.336133 \\ \mathrm{H} & 4.123101 & -0.527314 & 3.153456 \\ \mathrm{H} & 5.368164 & -2.551650 & 2.399131\end{array}$




$\begin{array}{lrrl}\mathrm{H} & 4.024496 & -4.551066 & 1.575401 \\ \mathrm{H} & 1.555520 & -4.397179 & 1.545764 \\ \mathrm{C} & -4.278191 & -0.965437 & 2.156898 \\ \mathrm{C} & -5.037068 & -2.159073 & 2.185273 \\ \mathrm{C} & -4.370163 & -3.363026 & 2.265189 \\ \mathrm{C} & -2.963207 & -3.387262 & 2.354682 \\ \mathrm{~N} & -2.227370 & -2.219946 & 2.342087 \\ \mathrm{C} & -2.902362 & -1.050118 & 2.224824 \\ \mathrm{C} & -2.167378 & -4.558230 & 2.539060 \\ \mathrm{C} & -2.612896 & -5.890504 & 2.410151 \\ \mathrm{C} & -1.769058 & -6.935210 & 2.714935 \\ \mathrm{C} & -0.462960 & -6.634908 & 3.171470 \\ \mathrm{C} & -0.072430 & -5.316904 & 3.245741 \\ \mathrm{~N} & -0.871358 & -4.277768 & 2.916528 \\ \mathrm{H} & -4.742493 & 0.018738 & 2.066624 \\ \mathrm{H} & -6.128841 & -2.125753 & 2.164222 \\ \mathrm{H} & -4.922814 & -4.303515 & 2.310112 \\ \mathrm{H} & -2.299848 & -0.141673 & 2.160980 \\ \mathrm{H} & -3.630204 & -6.082029 & 2.063009 \\ \mathrm{H} & -2.103409 & -7.970168 & 2.617011 \\ \mathrm{H} & 0.225019 & -7.421647 & 3.486051 \\ \mathrm{H} & 0.921984 & -5.052484 & 3.613862 \\ \mathrm{Ni} & -0.395022 & -2.440237 & 2.785579\end{array}$

\begin{tabular}{lrrr}
\multicolumn{4}{c}{ Cartesian coordinates of COF- $\mathbf{L}_{2} \mathbf{N i}^{2+}$ complex with COF data omitted } \\
$\mathrm{C}$ & -1.996911 & 5.302583 & 0.066865 \\
$\mathrm{C}$ & -0.620655 & 5.514549 & 0.049497 \\
$\mathrm{C}$ & 0.205113 & 4.648034 & 0.756446 \\
$\mathrm{C}$ & -0.371348 & 3.602602 & 1.469532 \\
$\mathrm{~N}$ & -1.711517 & 3.423407 & 1.509437 \\
$\mathrm{C}$ & -2.503188 & 4.249810 & 0.817709 \\
$\mathrm{C}$ & 0.377173 & 2.627539 & 2.262826 \\
$\mathrm{C}$ & 1.742114 & 2.386727 & 2.157788 \\
$\mathrm{C}$ & 2.292536 & 1.350243 & 2.902922 \\
$\mathrm{C}$ & 1.465921 & 0.563935 & 3.706008 \\
$\mathrm{C}$ & 0.112659 & 0.869305 & 3.766411 \\
$\mathrm{~N}$ & -0.407230 & 1.899403 & 3.085054 \\
$\mathrm{H}$ & -2.682134 & 5.952469 & -0.479335 \\
$\mathrm{H}$ & -0.192704 & 6.345606 & -0.515397 \\
$\mathrm{H}$ & 1.288485 & 4.771008 & 0.755054 \\
$\mathrm{H}$ & -3.576771 & 4.074732 & 0.877069 \\
$\mathrm{H}$ & 2.382436 & 2.915144 & 1.447480 \\
$\mathrm{H}$ & 3.364402 & 1.157715 & 2.818679 \\
$\mathrm{H}$ & 1.855350 & -0.280077 & 4.278082
\end{tabular}




$\begin{array}{llll}\mathrm{H} & -0.580368 & 0.280698 & 4.371895 \\ \mathrm{C} & -6.026964 & 1.728428 & 1.240965 \\ \mathrm{C} & -6.839630 & 1.695848 & 2.369375 \\ \mathrm{C} & -6.264140 & 1.823189 & 3.634313 \\ \mathrm{C} & -4.891575 & 2.018699 & 3.728736 \\ \mathrm{~N} & -4.120521 & 2.123933 & 2.620308 \\ \mathrm{C} & -4.658159 & 1.944001 & 1.407711 \\ \mathrm{C} & -4.123405 & 2.074823 & 4.975471 \\ \mathrm{C} & -4.674433 & 2.167297 & 6.251728 \\ \mathrm{C} & -3.827723 & 2.253968 & 7.352249 \\ \mathrm{C} & -2.450303 & 2.263892 & 7.145501 \\ \mathrm{C} & -1.969468 & 2.166172 & 5.846146 \\ \mathrm{~N} & -2.781720 & 2.045706 & 4.787824 \\ \mathrm{H} & -6.405742 & 1.560304 & 0.222360 \\ \mathrm{H} & -7.917056 & 1.542590 & 2.271537 \\ \mathrm{H} & -6.875738 & 1.747036 & 4.534175 \\ \mathrm{H} & -3.980189 & 1.920799 & 0.538806 \\ \mathrm{H} & -5.756758 & 2.193362 & 6.381606 \\ \mathrm{H} & -4.240723 & 2.331775 & 8.360505 \\ \mathrm{H} & -1.748562 & 2.355643 & 7.976043 \\ \mathrm{H} & -0.898242 & 2.195362 & 5.644676 \\ \mathrm{Ni} & -2.260305 & 2.278677 & 2.949757\end{array}$

Cartesian coordinates of $\mathrm{COFL}_{2} \mathrm{Ni}-\mathrm{CO} 2$ complex with $\mathrm{COF}$ data omitted

$\begin{array}{lrrr}\mathrm{C} & 1.007931 & 1.491096 & -4.687198 \\ \mathrm{C} & 1.750385 & 1.212474 & -3.545958 \\ \mathrm{C} & 1.515685 & 1.980123 & -2.408523 \\ \mathrm{C} & 0.536935 & 2.969877 & -2.461765 \\ \mathrm{~N} & -0.177618 & 3.241841 & -3.558781 \\ \mathrm{C} & 0.061576 & 2.516224 & -4.643433 \\ \mathrm{C} & 0.157195 & 3.746445 & -1.260513 \\ \mathrm{C} & 1.095663 & 4.405533 & -0.463957 \\ \mathrm{C} & 0.665108 & 5.158335 & 0.623105 \\ \mathrm{C} & -0.702216 & 5.221585 & 0.886746 \\ \mathrm{C} & -1.573489 & 4.515821 & 0.067121 \\ \mathrm{~N} & -1.161521 & 3.792499 & -0.983413 \\ \mathrm{H} & 1.141666 & 0.909585 & -5.601653 \\ \mathrm{H} & 2.491534 & 0.411839 & -3.501798 \\ \mathrm{H} & 2.041691 & 1.760474 & -1.477701 \\ \mathrm{H} & -0.544527 & 2.750289 & -5.525513 \\ \mathrm{H} & 2.152208 & 4.341106 & -0.730109 \\ \mathrm{H} & 1.382783 & 5.690879 & 1.251577 \\ \mathrm{H} & -1.096249 & 5.798287 & 1.725629 \\ \mathrm{H} & -2.649565 & 4.513965 & 0.255100\end{array}$




$\begin{array}{llll}\mathrm{C} & -5.906567 & 0.289242 & -2.119935 \\ \mathrm{C} & -6.911320 & 1.211732 & -2.420442 \\ \mathrm{C} & -6.559944 & 2.539857 & -2.621457 \\ \mathrm{C} & -5.221656 & 2.921458 & -2.486522 \\ \mathrm{~N} & -4.254505 & 2.032466 & -2.140240 \\ \mathrm{C} & -4.597009 & 0.738661 & -1.997355 \\ \mathrm{C} & -4.733974 & 4.280435 & -2.749238 \\ \mathrm{C} & -5.542651 & 5.403481 & -2.963006 \\ \mathrm{C} & -4.945029 & 6.619623 & -3.268185 \\ \mathrm{C} & -3.550374 & 6.688924 & -3.359651 \\ \mathrm{C} & -2.812516 & 5.540444 & -3.111557 \\ \mathrm{~N} & -3.387447 & 4.373781 & -2.799795 \\ \mathrm{H} & -6.114081 & -0.773500 & -1.970540 \\ \mathrm{H} & -7.951113 & 0.891805 & -2.521153 \\ \mathrm{H} & -7.312620 & 3.276377 & -2.906944 \\ \mathrm{H} & -3.792135 & 0.027287 & -1.793048 \\ \mathrm{H} & -6.628166 & 5.324569 & -2.888029 \\ \mathrm{H} & -5.559061 & 7.506647 & -3.440035 \\ \mathrm{H} & -3.044093 & 7.618688 & -3.625990 \\ \mathrm{H} & -1.720269 & 5.513398 & -3.177427 \\ \mathrm{Ni} & -2.479353 & 2.819765 & -1.986450 \\ \mathrm{C} & -1.704573 & 1.196557 & -1.426605 \\ \mathrm{O} & -1.549641 & 0.752846 & -2.556141 \\ \mathrm{O} & -1.470888 & 0.856035 & -0.268745\end{array}$

\section{Cartesian coordinates of $\mathrm{COF} \mathrm{L}_{\mathbf{2}} \mathrm{Ni}-\mathrm{H}_{\mathbf{2}} \mathrm{O}$ complex with $\mathrm{COF}$ data omitted}

$\begin{array}{lrrr}\mathrm{C} & -2.359920 & 1.632747 & 4.857013 \\ \mathrm{C} & -1.021672 & 2.051554 & 4.732832 \\ \mathrm{C} & -0.091971 & 1.166033 & 4.227136 \\ \mathrm{C} & -0.495461 & -0.125970 & 3.854332 \\ \mathrm{~N} & -1.797831 & -0.522411 & 3.975916 \\ \mathrm{C} & -2.697000 & 0.353994 & 4.468284 \\ \mathrm{C} & 0.367999 & -1.144014 & 3.313200 \\ \mathrm{C} & 1.747952 & -1.005319 & 3.094811 \\ \mathrm{C} & 2.462493 & -2.055739 & 2.551782 \\ \mathrm{C} & 1.775834 & -3.238780 & 2.210521 \\ \mathrm{C} & 0.413382 & -3.302874 & 2.420249 \\ \mathrm{~N} & -0.295727 & -2.292287 & 2.961466 \\ \mathrm{H} & -3.128506 & 2.293590 & 5.261181 \\ \mathrm{H} & -0.725161 & 3.062296 & 5.021164 \\ \mathrm{H} & 0.946675 & 1.461015 & 4.085243 \\ \mathrm{H} & -3.722056 & -0.012809 & 4.554656 \\ \mathrm{H} & 2.258114 & -0.067104 & 3.313326 \\ \mathrm{H} & 3.538740 & -1.948610 & 2.397411\end{array}$




$\begin{array}{lrrl}\mathrm{H} & 2.288950 & -4.091908 & 1.761483 \\ \mathrm{H} & -0.160845 & -4.186278 & 2.129147 \\ \mathrm{C} & -5.698386 & -0.606018 & 1.586019 \\ \mathrm{C} & -6.509121 & -1.761396 & 1.486292 \\ \mathrm{C} & -5.967527 & -2.986079 & 1.818125 \\ \mathrm{C} & -4.635449 & -3.067318 & 2.271197 \\ \mathrm{~N} & -3.863884 & -1.933355 & 2.394118 \\ \mathrm{C} & -4.402674 & -0.743406 & 2.035033 \\ \mathrm{C} & -3.949201 & -4.261151 & 2.662187 \\ \mathrm{C} & -4.404201 & -5.579831 & 2.467644 \\ \mathrm{C} & -3.647841 & -6.646435 & 2.904895 \\ \mathrm{C} & -2.415237 & -6.381563 & 3.548376 \\ \mathrm{C} & -2.014453 & -5.074316 & 3.705700 \\ \mathrm{~N} & -2.741962 & -4.015208 & 3.282797 \\ \mathrm{H} & -6.059288 & 0.393117 & 1.331060 \\ \mathrm{H} & -7.551233 & -1.685724 & 1.165886 \\ \mathrm{H} & -6.570878 & -3.894444 & 1.764305 \\ \mathrm{H} & -3.748037 & 0.130054 & 2.085692 \\ \mathrm{H} & -5.353834 & -5.746706 & 1.955372 \\ \mathrm{H} & -3.991723 & -7.672144 & 2.756948 \\ \mathrm{H} & -1.795356 & -7.189019 & 3.942391 \\ \mathrm{H} & -1.082119 & -4.828368 & 4.220694 \\ \mathrm{Ni} & -2.184085 & -2.217518 & 3.215201 \\ \mathrm{O} & -1.382967 & -0.590318 & 0.651001 \\ \mathrm{H} & -1.239175 & 0.253105 & 1.109951 \\ \mathrm{H} & -1.631306 & -1.205774 & 1.363570\end{array}$

\section{Cartesian coordinates of $\mathrm{L}_{2} \mathrm{Ni}-\mathrm{COF}-\mathrm{CO}_{2} \mathrm{H}^{+}$complex with $\mathrm{COF}$ data omitted}

$\begin{array}{lrrr}\mathrm{C} & 0.881393 & 2.022841 & -4.573378 \\ \mathrm{C} & 1.785471 & 1.734513 & -3.556782 \\ \mathrm{C} & 1.613575 & 2.359557 & -2.324011 \\ \mathrm{C} & 0.531134 & 3.222204 & -2.158407 \\ \mathrm{~N} & -0.354768 & 3.481353 & -3.128218 \\ \mathrm{C} & -0.172398 & 2.900573 & -4.307463 \\ \mathrm{C} & 0.224440 & 3.833771 & -0.848439 \\ \mathrm{C} & 1.211943 & 4.284884 & 0.031755 \\ \mathrm{C} & 0.840347 & 4.831651 & 1.253892 \\ \mathrm{C} & -0.514153 & 4.914861 & 1.572105 \\ \mathrm{C} & -1.439409 & 4.440998 & 0.651390 \\ \mathrm{~N} & -1.079741 & 3.921387 & -0.526627 \\ \mathrm{H} & 0.970885 & 1.559149 & -5.557779 \\ \mathrm{H} & 2.606393 & 1.021679 & -3.668839 \\ \mathrm{H} & 2.282563 & 2.113748 & -1.498380 \\ \mathrm{H} & -0.913706 & 3.128921 & -5.080475\end{array}$




$\begin{array}{lrrr}\mathrm{H} & 2.259976 & 4.220982 & -0.264353 \\ \mathrm{H} & 1.599757 & 5.193284 & 1.950937 \\ \mathrm{H} & -0.855192 & 5.335465 & 2.519362 \\ \mathrm{H} & -2.511260 & 4.468119 & 0.861904 \\ \mathrm{C} & -5.789393 & 0.614150 & -2.290037 \\ \mathrm{C} & -6.754877 & 1.548144 & -2.646681 \\ \mathrm{C} & -6.382905 & 2.883252 & -2.789321 \\ \mathrm{C} & -5.063022 & 3.251125 & -2.542310 \\ \mathrm{~N} & -4.126850 & 2.339730 & -2.177192 \\ \mathrm{C} & -4.481251 & 1.049879 & -2.079675 \\ \mathrm{C} & -4.560533 & 4.631190 & -2.658263 \\ \mathrm{C} & -5.277831 & 5.702544 & -3.194405 \\ \mathrm{C} & -4.658725 & 6.944451 & -3.292155 \\ \mathrm{C} & -3.339308 & 7.084251 & -2.865525 \\ \mathrm{C} & -2.692293 & 5.971461 & -2.340712 \\ \mathrm{~N} & -3.295294 & 4.788186 & -2.227822 \\ \mathrm{H} & -6.024660 & -0.445119 & -2.135928 \\ \mathrm{H} & -7.790636 & 1.243840 & -2.813625 \\ \mathrm{H} & -7.119045 & 3.635598 & -3.074161 \\ \mathrm{H} & -3.707523 & 0.317248 & -1.838496 \\ \mathrm{H} & -6.300163 & 5.565819 & -3.547286 \\ \mathrm{H} & -5.200037 & 7.795190 & -3.712026 \\ \mathrm{H} & -2.813437 & 8.037111 & -2.942580 \\ \mathrm{H} & -1.652438 & 6.011908 & -2.009565 \\ \mathrm{Ni} & -2.467455 & 3.112835 & -1.611368 \\ \mathrm{C} & -1.698645 & 1.468467 & -1.179025 \\ \mathrm{O} & -1.401085 & 0.832747 & -2.290092 \\ \mathrm{O} & -1.421467 & 1.060334 & -0.062240 \\ \mathrm{H} & -0.780456 & 0.061936 & -2.106876\end{array}$

\section{Cartesian coordinates of $\mathrm{COF}-\mathrm{L}_{2} \mathrm{NiH}^{+}$complex with $\mathrm{COF}$ data omitted}

$\begin{array}{lrrr}\mathrm{C} & -0.110481 & -1.167374 & -4.916477 \\ \mathrm{C} & 1.225500 & -1.513428 & -4.735959 \\ \mathrm{C} & 2.061581 & -0.652359 & -4.035287 \\ \mathrm{C} & 1.530476 & 0.546551 & -3.555487 \\ \mathrm{~N} & 0.248740 & 0.881276 & -3.750623 \\ \mathrm{C} & -0.555364 & 0.041816 & -4.389434 \\ \mathrm{C} & 2.329619 & 1.543845 & -2.814628 \\ \mathrm{C} & 3.726384 & 1.497142 & -2.716419 \\ \mathrm{C} & 4.401868 & 2.521580 & -2.069999 \\ \mathrm{C} & 3.672240 & 3.575877 & -1.517241 \\ \mathrm{C} & 2.288656 & 3.537419 & -1.608185 \\ \mathrm{~N} & 1.637645 & 2.552275 & -2.240227 \\ \mathrm{H} & -0.803873 & -1.822026 & -5.447601\end{array}$




$\begin{array}{lrrl}\mathrm{H} & 1.613011 & -2.460158 & -5.119242 \\ \mathrm{H} & 3.090558 & -0.926614 & -3.811414 \\ \mathrm{H} & -1.602126 & 0.346543 & -4.490324 \\ \mathrm{H} & 4.278477 & 0.652685 & -3.124307 \\ \mathrm{H} & 5.491467 & 2.490485 & -1.997513 \\ \mathrm{H} & 4.161795 & 4.404071 & -1.002024 \\ \mathrm{H} & 1.664689 & 4.311547 & -1.155909 \\ \mathrm{C} & -4.249191 & 1.424305 & -2.228401 \\ \mathrm{C} & -4.885143 & 2.540856 & -2.768195 \\ \mathrm{C} & -4.115142 & 3.622536 & -3.185559 \\ \mathrm{C} & -2.729548 & 3.570910 & -3.031336 \\ \mathrm{~N} & -2.129461 & 2.515706 & -2.431603 \\ \mathrm{C} & -2.866223 & 1.456972 & -2.067794 \\ \mathrm{C} & -1.786343 & 4.559804 & -3.568590 \\ \mathrm{C} & -2.150851 & 5.766111 & -4.170653 \\ \mathrm{C} & -1.161598 & 6.580035 & -4.709519 \\ \mathrm{C} & 0.166993 & 6.160758 & -4.643942 \\ \mathrm{C} & 0.453144 & 4.953341 & -4.019977 \\ \mathrm{~N} & -0.492093 & 4.177591 & -3.481074 \\ \mathrm{H} & -4.788567 & 0.514841 & -1.950283 \\ \mathrm{H} & -5.969706 & 2.553042 & -2.897798 \\ \mathrm{H} & -4.583572 & 4.483643 & -3.663461 \\ \mathrm{H} & -2.327886 & 0.597385 & -1.664525 \\ \mathrm{H} & -3.198654 & 6.064408 & -4.217681 \\ \mathrm{H} & -1.424929 & 7.527989 & -5.183430 \\ \mathrm{H} & 0.974092 & 6.757366 & -5.072166 \\ \mathrm{H} & 1.478858 & 4.584042 & -3.947817 \\ \mathrm{Ni} & -0.256385 & 2.537459 & -2.404458 \\ \mathrm{H} & -0.243278 & 1.676239 & -1.232018\end{array}$




\begin{tabular}{|c|c|c|c|}
\hline \multicolumn{4}{|c|}{ Cartesian coordinates of $\mathrm{L}_{2} \mathrm{Co}^{+1}$} \\
\hline $\mathrm{C}$ & 1.014087 & 3.803599 & 1.594021 \\
\hline $\mathrm{C}$ & 2.396698 & 3.820735 & 1.410760 \\
\hline $\mathrm{C}$ & 3.005419 & 2.721254 & 0.814515 \\
\hline $\mathrm{C}$ & 2.218132 & 1.647197 & 0.404188 \\
\hline $\mathrm{N}$ & 0.867882 & 1.652749 & 0.547040 \\
\hline $\mathrm{C}$ & 0.291486 & 2.703890 & 1.152235 \\
\hline $\mathrm{C}$ & 2.718616 & 0.398922 & -0.173093 \\
\hline $\mathrm{C}$ & 4.008756 & 0.191832 & -0.657277 \\
\hline $\mathrm{C}$ & 4.326359 & -1.029130 & -1.243187 \\
\hline $\mathrm{C}$ & 3.333198 & -2.003484 & -1.342012 \\
\hline $\mathrm{C}$ & 2.072129 & -1.731638 & -0.829670 \\
\hline $\mathrm{N}$ & 1.766744 & -0.567411 & -0.234096 \\
\hline $\mathrm{H}$ & 0.494851 & 4.632721 & 2.077528 \\
\hline $\mathrm{H}$ & 2.994535 & 4.671313 & 1.744060 \\
\hline $\mathrm{H}$ & 4.088433 & 2.687128 & 0.689279 \\
\hline $\mathrm{H}$ & -0.789978 & 2.647126 & 1.288930 \\
\hline $\mathrm{H}$ & 4.751892 & 0.987999 & -0.596974 \\
\hline $\mathrm{H}$ & 5.329577 & -1.211534 & -1.633399 \\
\hline $\mathrm{H}$ & 3.528478 & -2.967790 & -1.814038 \\
\hline $\mathrm{H}$ & 1.264865 & -2.463050 & -0.899185 \\
\hline $\mathrm{C}$ & -3.391110 & 1.911654 & -1.287109 \\
\hline $\mathrm{C}$ & -4.376930 & 0.945429 & -1.086952 \\
\hline $\mathrm{C}$ & -4.029627 & -0.241828 & -0.450784 \\
\hline $\mathrm{C}$ & -2.717309 & -0.423536 & -0.018830 \\
\hline $\mathrm{N}$ & -1.770888 & 0.536820 & -0.178818 \\
\hline $\mathrm{C}$ & -2.106372 & 1.666872 & -0.822111 \\
\hline $\mathrm{C}$ & -2.187727 & -1.638763 & 0.601402 \\
\hline $\mathrm{C}$ & -2.952715 & -2.687641 & 1.107792 \\
\hline $\mathrm{C}$ & -2.314308 & -3.753365 & 1.733556 \\
\hline $\mathrm{C}$ & -0.924484 & -3.727844 & 1.848582 \\
\hline $\mathrm{C}$ & -0.225661 & -2.655008 & 1.312522 \\
\hline $\mathrm{N}$ & -0.832147 & -1.638091 & 0.678930 \\
\hline $\mathrm{H}$ & -3.610345 & 2.848761 & -1.801496 \\
\hline $\mathrm{H}$ & -5.398088 & 1.107294 & -1.437693 \\
\hline $\mathrm{H}$ & -4.767829 & -1.032571 & -0.310947 \\
\hline $\mathrm{H}$ & -1.304464 & 2.392358 & -0.970719 \\
\hline $\mathrm{H}$ & -4.040563 & -2.659148 & 1.033336 \\
\hline $\mathrm{H}$ & -2.894115 & -4.583579 & 2.141661 \\
\hline $\mathrm{H}$ & -0.381289 & -4.529715 & 2.351344 \\
\hline $\mathrm{H}$ & 0.861064 & -2.592128 & 1.393449 \\
\hline Co & 0.007874 & -0.003940 & 0.203145 \\
\hline
\end{tabular}

Cartesian coordinates of $\mathrm{L}_{2} \mathrm{Co}-\mathrm{CO}_{2}{ }^{1+}$

$\begin{array}{llll}\mathrm{C} & 0.738811 & 3.188089 & 2.226854\end{array}$ 


\begin{tabular}{|c|c|c|c|}
\hline $\mathrm{C}$ & 2.111486 & 3.259494 & 1.990538 \\
\hline $\mathrm{C}$ & 2.744499 & 2.178944 & 1.384962 \\
\hline $\mathrm{C}$ & 1.988568 & 1.067494 & 1.017486 \\
\hline $\mathrm{N}$ & 0.646990 & 1.022649 & 1.208650 \\
\hline $\mathrm{C}$ & 0.047294 & 2.054217 & 1.822452 \\
\hline $\mathrm{C}$ & 2.518703 & -0.169200 & 0.441941 \\
\hline $\mathrm{C}$ & 3.802577 & -0.329921 & -0.074523 \\
\hline $\mathrm{C}$ & 4.157319 & -1.545674 & -0.649294 \\
\hline $\mathrm{C}$ & 3.204672 & -2.563119 & -0.702195 \\
\hline $\mathrm{C}$ & 1.946506 & -2.336269 & -0.162342 \\
\hline $\mathrm{N}$ & 1.604237 & -1.174301 & 0.421279 \\
\hline $\mathrm{H}$ & 0.204033 & 4.001067 & 2.720627 \\
\hline $\mathrm{H}$ & 2.684845 & 4.138823 & 2.290748 \\
\hline $\mathrm{H}$ & 3.822464 & 2.189814 & 1.219482 \\
\hline $\mathrm{H}$ & -1.026195 & 1.953348 & 1.994974 \\
\hline $\mathrm{H}$ & 4.511364 & 0.498621 & -0.048357 \\
\hline $\mathrm{H}$ & 5.156217 & -1.691702 & -1.064938 \\
\hline $\mathrm{H}$ & 3.427869 & -3.527223 & -1.162080 \\
\hline $\mathrm{H}$ & 1.169283 & -3.100541 & -0.208644 \\
\hline $\mathrm{C}$ & -3.515350 & 1.221521 & -0.795124 \\
\hline $\mathrm{C}$ & -4.469154 & 0.205297 & -0.739767 \\
\hline $\mathrm{C}$ & -4.124794 & -0.999631 & -0.136582 \\
\hline $\mathrm{C}$ & -2.850018 & -1.150867 & 0.404679 \\
\hline $\mathrm{N}$ & -1.934915 & -0.146410 & 0.381050 \\
\hline $\mathrm{C}$ & -2.266696 & 1.004602 & -0.229693 \\
\hline $\mathrm{C}$ & -2.330310 & -2.376722 & 1.012023 \\
\hline $\mathrm{C}$ & -3.092911 & -3.481054 & 1.387099 \\
\hline $\mathrm{C}$ & -2.470812 & -4.550332 & 2.023216 \\
\hline $\mathrm{C}$ & -1.102320 & -4.474779 & 2.281478 \\
\hline $\mathrm{C}$ & -0.403492 & -3.348694 & 1.868000 \\
\hline $\mathrm{N}$ & -0.992154 & -2.328528 & 1.225132 \\
\hline $\mathrm{H}$ & -3.730253 & 2.176987 & -1.276486 \\
\hline $\mathrm{H}$ & -5.460732 & 0.343680 & -1.175089 \\
\hline $\mathrm{H}$ & -4.834267 & -1.827478 & -0.107207 \\
\hline $\mathrm{H}$ & -1.488577 & 1.767925 & -0.276682 \\
\hline $\mathrm{H}$ & -4.167914 & -3.494785 & 1.203552 \\
\hline $\mathrm{H}$ & -3.049618 & -5.423850 & 2.329868 \\
\hline $\mathrm{H}$ & -0.576386 & -5.278569 & 2.799213 \\
\hline $\mathrm{H}$ & 0.666950 & -3.244844 & 2.056904 \\
\hline $\mathrm{Co}$ & -0.169402 & -0.656223 & 0.864979 \\
\hline $\mathrm{C}$ & -0.142067 & -0.685011 & -2.284442 \\
\hline $\mathrm{O}$ & 0.317157 & 0.380262 & -2.300250 \\
\hline $\mathrm{O}$ & -0.601136 & -1.750456 & -2.288452 \\
\hline
\end{tabular}

Cartesian coordinates of $\mathrm{L}_{2} \mathrm{Co}-\mathrm{H}_{2} \mathrm{O}^{1+}$ 


\begin{tabular}{|c|c|c|c|}
\hline $\mathrm{C}$ & -3.635804 & 3.252747 & -2.138253 \\
\hline $\mathrm{C}$ & -4.901460 & 2.694892 & -1.965938 \\
\hline $\mathrm{C}$ & -5.011017 & 1.467902 & -1.320018 \\
\hline $\mathrm{C}$ & -3.858159 & 0.843145 & -0.851945 \\
\hline $\mathrm{N}$ & -2.634196 & 1.405656 & -0.993841 \\
\hline $\mathrm{C}$ & -2.531096 & 2.575482 & -1.639730 \\
\hline $\mathrm{C}$ & -3.806506 & -0.465708 & -0.199288 \\
\hline $\mathrm{C}$ & -4.907827 & -1.171310 & 0.279955 \\
\hline $\mathrm{C}$ & -4.707222 & -2.376278 & 0.944595 \\
\hline $\mathrm{C}$ & -3.401572 & -2.833537 & 1.123703 \\
\hline $\mathrm{C}$ & -2.352071 & -2.084113 & 0.611459 \\
\hline $\mathrm{N}$ & -2.539108 & -0.934287 & -0.058257 \\
\hline $\mathrm{H}$ & -3.499644 & 4.204722 & -2.653815 \\
\hline $\mathrm{H}$ & -5.791657 & 3.202943 & -2.342101 \\
\hline $\mathrm{H}$ & -5.984238 & 0.991947 & -1.195411 \\
\hline $\mathrm{H}$ & -1.522092 & 2.973904 & -1.759248 \\
\hline $\mathrm{H}$ & -5.913086 & -0.767345 & 0.155406 \\
\hline $\mathrm{H}$ & -5.556196 & -2.942402 & 1.332859 \\
\hline $\mathrm{H}$ & -3.192814 & -3.762306 & 1.657237 \\
\hline $\mathrm{H}$ & -1.315965 & -2.402036 & 0.742225 \\
\hline $\mathrm{C}$ & 0.947478 & 3.630959 & 0.891911 \\
\hline $\mathrm{C}$ & 2.270646 & 3.220828 & 0.730986 \\
\hline $\mathrm{C}$ & 2.520306 & 1.968438 & 0.178994 \\
\hline $\mathrm{C}$ & 1.445247 & 1.176794 & -0.219887 \\
\hline $\mathrm{N}$ & 0.161906 & 1.603267 & -0.108119 \\
\hline $\mathrm{C}$ & -0.076450 & 2.795188 & 0.465855 \\
\hline $\mathrm{C}$ & 1.543423 & -0.185685 & -0.747822 \\
\hline $\mathrm{C}$ & 2.716538 & -0.801012 & -1.179847 \\
\hline $\mathrm{C}$ & 2.653369 & -2.081470 & -1.720121 \\
\hline $\mathrm{C}$ & 1.409781 & -2.702699 & -1.828849 \\
\hline $\mathrm{C}$ & 0.283377 & -2.033759 & -1.369193 \\
\hline $\mathrm{N}$ & 0.340546 & -0.812394 & -0.814945 \\
\hline $\mathrm{H}$ & 0.702421 & 4.590845 & 1.349614 \\
\hline $\mathrm{H}$ & 3.096985 & 3.858155 & 1.052142 \\
\hline $\mathrm{H}$ & 3.541952 & 1.599884 & 0.078523 \\
\hline $\mathrm{H}$ & -1.126712 & 3.051949 & 0.623656 \\
\hline $\mathrm{H}$ & 3.668708 & -0.272854 & -1.116130 \\
\hline $\mathrm{H}$ & 3.559812 & -2.580010 & -2.069267 \\
\hline $\mathrm{H}$ & 1.307112 & -3.696411 & -2.267911 \\
\hline $\mathrm{H}$ & -0.709319 & -2.480509 & -1.449351 \\
\hline $\mathrm{Co}$ & -1.165784 & 0.304085 & -0.504996 \\
\hline $\mathrm{O}$ & -2.808984 & 1.832524 & 1.881712 \\
\hline $\mathrm{H}$ & -2.682864 & 1.770577 & 2.834855 \\
\hline $\mathrm{H}$ & -2.229750 & 1.145859 & 1.519413 \\
\hline
\end{tabular}




\begin{tabular}{|c|c|c|c|}
\hline \multicolumn{4}{|c|}{ Cartesian coordinates of $\mathrm{L}_{2} \mathrm{CoH}^{+}$} \\
\hline $\mathrm{C}$ & -2.367646 & -3.182637 & 1.270523 \\
\hline $\mathrm{C}$ & -3.647659 & -2.643481 & 1.172505 \\
\hline $\mathrm{C}$ & -3.804285 & -1.368605 & 0.633183 \\
\hline $\mathrm{C}$ & -2.681775 & -0.679320 & 0.183497 \\
\hline $\mathrm{N}$ & -1.446343 & -1.233783 & 0.231373 \\
\hline $\mathrm{C}$ & -1.293570 & -2.442314 & 0.792102 \\
\hline $\mathrm{C}$ & -2.692921 & 0.692620 & -0.337465 \\
\hline $\mathrm{C}$ & -3.838256 & 1.402812 & -0.687771 \\
\hline $\mathrm{C}$ & -3.705604 & 2.675116 & -1.238365 \\
\hline $\mathrm{C}$ & -2.428468 & 3.189889 & -1.447399 \\
\hline $\mathrm{C}$ & -1.330792 & 2.427649 & -1.067982 \\
\hline $\mathrm{N}$ & -1.458511 & 1.225434 & -0.489411 \\
\hline $\mathrm{H}$ & -2.193671 & -4.163828 & 1.715864 \\
\hline $\mathrm{H}$ & -4.516495 & -3.199919 & 1.532028 \\
\hline $\mathrm{H}$ & -4.791705 & -0.908039 & 0.586829 \\
\hline $\mathrm{H}$ & -0.275890 & -2.826452 & 0.872229 \\
\hline $\mathrm{H}$ & -4.826135 & 0.960048 & -0.556162 \\
\hline $\mathrm{H}$ & -4.591506 & 3.247146 & -1.523947 \\
\hline $\mathrm{H}$ & -2.275362 & 4.166222 & -1.910777 \\
\hline $\mathrm{H}$ & -0.314928 & 2.782073 & -1.249200 \\
\hline $\mathrm{C}$ & 2.421573 & -3.208343 & -1.444637 \\
\hline $\mathrm{C}$ & 3.698800 & -2.693641 & -1.235985 \\
\hline $\mathrm{C}$ & 3.831686 & -1.420792 & -0.686708 \\
\hline $\mathrm{C}$ & 2.686465 & -0.710014 & -0.337213 \\
\hline $\mathrm{N}$ & 1.451962 & -1.242805 & -0.488600 \\
\hline $\mathrm{C}$ & 1.324014 & -2.445521 & -1.066037 \\
\hline $\mathrm{C}$ & 2.675532 & 0.662512 & 0.182201 \\
\hline $\mathrm{C}$ & 3.798192 & 1.352400 & 0.630590 \\
\hline $\mathrm{C}$ & 3.641711 & 2.627813 & 1.168685 \\
\hline $\mathrm{C}$ & 2.361700 & 3.166958 & 1.266820 \\
\hline $\mathrm{C}$ & 1.287452 & 2.426087 & 0.789640 \\
\hline $\mathrm{N}$ & 1.440103 & 1.217005 & 0.230080 \\
\hline $\mathrm{H}$ & 2.268302 & -4.185085 & -1.907100 \\
\hline $\mathrm{H}$ & 4.584601 & -3.266155 & -1.520912 \\
\hline $\mathrm{H}$ & 4.819656 & -0.978110 & -0.555508 \\
\hline $\mathrm{H}$ & 0.308086 & -2.799855 & -1.247056 \\
\hline $\mathrm{H}$ & 4.785650 & 0.891923 & 0.584175 \\
\hline $\mathrm{H}$ & 4.510687 & 3.184706 & 1.527162 \\
\hline $\mathrm{H}$ & 2.187860 & 4.148571 & 1.711283 \\
\hline $\mathrm{H}$ & 0.269762 & 2.810213 & 0.869755 \\
\hline Co & -0.003028 & -0.008285 & -0.170260 \\
\hline $\mathrm{H}$ & -0.001500 & -0.007621 & -1.567888 \\
\hline
\end{tabular}

Cartesian coordinates of $\left[\mathrm{COF}-\mathrm{L}_{2} \mathrm{Co}-\mathrm{CO}_{2}\right]^{1+}$ complex with $\mathrm{COF}$ data omitted 


\begin{tabular}{|c|c|c|c|}
\hline $\mathrm{C}$ & -1.325106 & -1.226429 & -4.793404 \\
\hline $\mathrm{C}$ & -2.314726 & -1.335058 & -3.821460 \\
\hline $\mathrm{C}$ & -2.061943 & -2.111542 & -2.695059 \\
\hline $\mathrm{C}$ & -0.816152 & -2.723141 & -2.563234 \\
\hline $\mathrm{N}$ & 0.150786 & -2.576727 & -3.486228 \\
\hline $\mathrm{C}$ & -0.102870 & -1.856918 & -4.575386 \\
\hline $\mathrm{C}$ & -0.409226 & -3.503813 & -1.379348 \\
\hline $\mathrm{C}$ & -1.299315 & -4.159380 & -0.524163 \\
\hline $\mathrm{C}$ & -0.795668 & -4.866261 & 0.562191 \\
\hline $\mathrm{C}$ & 0.585033 & -4.896522 & 0.768525 \\
\hline $\mathrm{C}$ & 1.404813 & -4.218343 & -0.125627 \\
\hline $\mathrm{N}$ & 0.919540 & -3.549115 & -1.170928 \\
\hline $\mathrm{H}$ & -1.475958 & -0.625306 & -5.691666 \\
\hline $\mathrm{H}$ & -3.257780 & -0.790504 & -3.886032 \\
\hline $\mathrm{H}$ & -2.810172 & -2.175637 & -1.904945 \\
\hline $\mathrm{H}$ & 0.721648 & -1.753889 & -5.286288 \\
\hline $\mathrm{H}$ & -2.370516 & -4.133123 & -0.729649 \\
\hline $\mathrm{H}$ & -1.472053 & -5.392738 & 1.239323 \\
\hline $\mathrm{H}$ & 1.024399 & -5.439710 & 1.606958 \\
\hline $\mathrm{H}$ & 2.493070 & -4.207001 & -0.015677 \\
\hline $\mathrm{C}$ & 6.056950 & -0.977041 & -1.564198 \\
\hline $\mathrm{C}$ & 6.856634 & -2.139310 & -1.594204 \\
\hline $\mathrm{C}$ & 6.280964 & -3.346019 & -1.932405 \\
\hline $\mathrm{C}$ & 4.900381 & -3.411681 & -2.235511 \\
\hline $\mathrm{N}$ & 4.122880 & -2.285368 & -2.141182 \\
\hline $\mathrm{C}$ & 4.706461 & -1.111698 & -1.840446 \\
\hline $\mathrm{C}$ & 4.226383 & -4.600775 & -2.693387 \\
\hline $\mathrm{C}$ & 4.818975 & -5.882815 & -2.783192 \\
\hline $\mathrm{C}$ & 4.087234 & -6.953024 & -3.248709 \\
\hline $\mathrm{C}$ & 2.736425 & -6.754779 & -3.627848 \\
\hline $\mathrm{C}$ & 2.213959 & -5.482905 & -3.507559 \\
\hline $\mathrm{N}$ & 2.916046 & -4.429235 & -3.068494 \\
\hline $\mathrm{H}$ & 6.462230 & 0.019787 & -1.378509 \\
\hline $\mathrm{H}$ & 7.926959 & -2.083649 & -1.379121 \\
\hline $\mathrm{H}$ & 6.893484 & -4.246695 & -1.996590 \\
\hline $\mathrm{H}$ & 4.064245 & -0.225056 & -1.838344 \\
\hline $\mathrm{H}$ & 5.856760 & -6.023810 & -2.476687 \\
\hline $\mathrm{H}$ & 4.544301 & -7.942826 & -3.319700 \\
\hline $\mathrm{H}$ & 2.121309 & -7.572707 & -4.005755 \\
\hline $\mathrm{H}$ & 1.173464 & -5.268588 & -3.776041 \\
\hline Co & 2.115610 & -2.626346 & -2.539718 \\
\hline $\mathrm{C}$ & 1.704520 & -0.948102 & -1.549127 \\
\hline $\mathrm{O}$ & 1.792645 & -0.501664 & -2.670655 \\
\hline $\mathrm{O}$ & 1.512433 & -0.762517 & -0.379216 \\
\hline
\end{tabular}




\begin{tabular}{|c|c|c|c|}
\hline Car & ordinates of & $\mathrm{COF} \mathrm{L}_{2} \mathrm{Co}$ & $\mathrm{O}^{+}$comple \\
\hline $\mathrm{C}$ & -2.932202 & -0.781985 & 2.960526 \\
\hline $\mathrm{C}$ & -3.154752 & -0.137132 & 4.176112 \\
\hline $\mathrm{C}$ & -2.099711 & -0.018796 & 5.074101 \\
\hline $\mathrm{C}$ & -0.870123 & -0.593186 & 4.754584 \\
\hline $\mathrm{N}$ & -0.668761 & -1.257928 & 3.586974 \\
\hline $\mathrm{C}$ & -1.669616 & -1.299786 & 2.689866 \\
\hline $\mathrm{C}$ & 0.322028 & -0.552813 & 5.595897 \\
\hline $\mathrm{C}$ & 0.360581 & -0.086742 & 6.909776 \\
\hline $\mathrm{C}$ & 1.544172 & -0.170690 & 7.632220 \\
\hline $\mathrm{C}$ & 2.656641 & -0.745548 & 7.015856 \\
\hline $\mathrm{C}$ & 2.551613 & -1.183248 & 5.704956 \\
\hline $\mathrm{N}$ & 1.420967 & -1.070075 & 4.981017 \\
\hline $\mathrm{H}$ & -3.713830 & -0.936272 & 2.214832 \\
\hline $\mathrm{H}$ & -4.137390 & 0.272491 & 4.421010 \\
\hline $\mathrm{H}$ & -2.230074 & 0.507521 & 6.020386 \\
\hline $\mathrm{H}$ & -1.447742 & -1.751212 & 1.718597 \\
\hline $\mathrm{H}$ & -0.541196 & 0.318596 & 7.370079 \\
\hline $\mathrm{H}$ & 1.593510 & 0.184932 & 8.663117 \\
\hline $\mathrm{H}$ & 3.603482 & -0.861836 & 7.545866 \\
\hline $\mathrm{H}$ & 3.398292 & -1.653644 & 5.205453 \\
\hline $\mathrm{C}$ & 5.124351 & -1.378827 & 2.266859 \\
\hline $\mathrm{C}$ & 5.416308 & -2.567972 & 1.604141 \\
\hline $\mathrm{C}$ & 4.411902 & -3.517118 & 1.467327 \\
\hline $\mathrm{C}$ & 3.150338 & -3.252301 & 1.995643 \\
\hline $\mathrm{N}$ & 2.905250 & -2.141095 & 2.722065 \\
\hline $\mathrm{C}$ & 3.864728 & -1.210635 & 2.819820 \\
\hline $\mathrm{C}$ & 1.950682 & -4.066101 & 1.781615 \\
\hline $\mathrm{C}$ & 1.936804 & -5.301000 & 1.135586 \\
\hline $\mathrm{C}$ & 0.732823 & -5.981616 & 0.990704 \\
\hline $\mathrm{C}$ & -0.424776 & -5.405886 & 1.509397 \\
\hline $\mathrm{C}$ & -0.335280 & -4.171236 & 2.138622 \\
\hline $\mathrm{N}$ & 0.816142 & -3.498301 & 2.262809 \\
\hline $\mathrm{H}$ & 5.868665 & -0.585326 & 2.337696 \\
\hline $\mathrm{H}$ & 6.395241 & -2.703888 & 1.144540 \\
\hline $\mathrm{H}$ & 4.594586 & -4.435282 & 0.908422 \\
\hline $\mathrm{H}$ & 3.589512 & -0.294866 & 3.345609 \\
\hline $\mathrm{H}$ & 2.864817 & -5.729991 & 0.756717 \\
\hline $\mathrm{H}$ & 0.700674 & -6.949803 & 0.486950 \\
\hline $\mathrm{H}$ & -1.395048 & -5.898115 & 1.427754 \\
\hline $\mathrm{H}$ & -1.225252 & -3.697039 & 2.551248 \\
\hline $\mathrm{Co}$ & 1.104309 & -1.930781 & 3.336170 \\
\hline $\mathrm{O}$ & 1.570719 & 0.436007 & 1.438755 \\
\hline $\mathrm{H}$ & 1.803501 & -0.325137 & 0.877018 \\
\hline
\end{tabular}




\begin{tabular}{|c|c|c|c|}
\hline $\mathrm{H}$ & 1.24402 & $2 \quad-0.0075$ & $\begin{array}{ll}6 & 2.240557\end{array}$ \\
\hline Cart & n coordinates & of $\left[\mathrm{COF}-\mathrm{L}_{2}\right.$ & $\left.\mathrm{CoH}^{+}\right]^{+}$complex \\
\hline $\mathrm{C}$ & -2.894207 & -5.258978 & -1.119814 \\
\hline $\mathrm{C}$ & -4.200691 & -4.784387 & -1.233403 \\
\hline $\mathrm{C}$ & -4.616666 & -3.737470 & -0.419162 \\
\hline $\mathrm{C}$ & -3.719791 & -3.204763 & 0.502730 \\
\hline $\mathrm{N}$ & -2.471697 & -3.708500 & 0.629952 \\
\hline $\mathrm{C}$ & -2.052461 & -4.692111 & -0.171884 \\
\hline $\mathrm{C}$ & -4.011053 & -2.117811 & 1.436370 \\
\hline $\mathrm{C}$ & -5.166360 & -1.339118 & 1.450703 \\
\hline $\mathrm{C}$ & -5.278625 & -0.354764 & 2.424787 \\
\hline $\mathrm{C}$ & -4.245408 & -0.163683 & 3.345461 \\
\hline $\mathrm{C}$ & -3.115435 & -0.963987 & 3.250114 \\
\hline $\mathrm{N}$ & -3.013476 & -1.915112 & 2.315630 \\
\hline $\mathrm{H}$ & -2.523094 & -6.065393 & -1.754081 \\
\hline $\mathrm{H}$ & -4.886085 & -5.216383 & -1.965687 \\
\hline $\mathrm{H}$ & -5.617531 & -3.313244 & -0.505867 \\
\hline $\mathrm{H}$ & -1.022879 & -5.026223 & -0.038852 \\
\hline $\mathrm{H}$ & -5.926941 & -1.428912 & 0.669334 \\
\hline $\mathrm{H}$ & -6.174648 & 0.268947 & 2.458913 \\
\hline $\mathrm{H}$ & -4.308575 & 0.597487 & 4.124938 \\
\hline $\mathrm{H}$ & -2.270580 & -0.850931 & 3.936084 \\
\hline $\mathrm{C}$ & 2.536073 & -4.004223 & 1.332148 \\
\hline $\mathrm{C}$ & 2.707804 & -5.087926 & 2.184460 \\
\hline $\mathrm{C}$ & 1.618172 & -5.562266 & 2.919755 \\
\hline $\mathrm{C}$ & 0.384278 & -4.935937 & 2.787517 \\
\hline $\mathrm{N}$ & 0.220761 & -3.897490 & 1.926214 \\
\hline $\mathrm{C}$ & 1.264411 & -3.442038 & 1.215367 \\
\hline $\mathrm{C}$ & -0.815993 & -5.285231 & 3.571799 \\
\hline $\mathrm{C}$ & -0.928767 & -6.410799 & 4.388143 \\
\hline $\mathrm{C}$ & -2.114197 & -6.620005 & 5.088234 \\
\hline $\mathrm{C}$ & -3.156655 & -5.704820 & 4.955846 \\
\hline $\mathrm{C}$ & -2.971298 & -4.610453 & 4.118930 \\
\hline $\mathrm{N}$ & -1.833404 & -4.407400 & 3.452117 \\
\hline $\mathrm{H}$ & 3.345731 & -3.536545 & 0.758169 \\
\hline $\mathrm{H}$ & 3.687930 & -5.558019 & 2.295793 \\
\hline $\mathrm{H}$ & 1.743643 & -6.394559 & 3.613121 \\
\hline $\mathrm{H}$ & 1.065591 & -2.597575 & 0.538573 \\
\hline $\mathrm{H}$ & -0.107383 & -7.122940 & 4.471357 \\
\hline $\mathrm{H}$ & -2.223134 & -7.496372 & 5.731257 \\
\hline $\mathrm{H}$ & -4.100481 & -5.835273 & 5.487712 \\
\hline $\mathrm{H}$ & -3.759298 & -3.866321 & 3.974167 \\
\hline $\mathrm{Co}$ & -1.477428 & -2.991733 & 2.025354 \\
\hline $\mathrm{H}$ & -1.090253 & -2.040213 & 1.025732 \\
\hline
\end{tabular}




\section{Reference}

[1] D. B. Shinde, H. B. Aiyappa, M. Bhadra, B. P. Biswal, P. Wadge, S. Kandambeth, B. Garai, T. Kundu, S. Kurungot, R. Banerjee, J. Mater. Chem. 2016, 4, 2682-2690.

[2] J. H. Chong, S. Marc, B. O. Patrick, M. J. Maclachlan, Org. Lett. 2003, 5, 3823-3826.

[3] M. J. T. Frisch, G. W. H. B. Schlegel, G. E. Scuseria, M. A. Robb, J. R. Cheeseman, G. Scalmani, V.Barone, B. Mennucci, G. A. Petersson, H. Nakatsuji, M. Caricato, X. Li, H. P. Hratchian, A. F. Izmaylov,J. Bloino, G. Zheng, J. L. Sonnenberg, M. Hada, M. Ehara, K. Toyota, R. Fukuda, J. Hasegawa, M. Ishida,T. Nakajima, Y. Honda, O. Kitao, H. Nakai, T. Vreven, J. A. Montgomery, Jr., J. E. Peralta, F. Ogliaro, M.Bearpark, J. J. Heyd, E. Brothers, K. N. Kudin, V. N. Staroverov, R. Kobayashi, J. Normand, K.Raghavachari, A. Rendell, J. C. Burant, S. S. Iyengar, J. Tomasi, M. Cossi, N. Rega, J. M. Millam, M.Klene, J. E. Knox, J. B. Cross, V. Bakken, C. Adamo, J. Jaramillo, R. Gomperts, R. E. Stratmann, O.Yazyev, A. J. Austin, R. Cammi, C. Pomelli, J. W. Ochterski, R. L. Martin, K. Morokuma, V. G.Zakrzewski, G. A. Voth, P. Salvador, J. J. Dannenberg, S. Dapprich, A. D. Daniels, Ö. Farkas, J. B.Foresman, J. V. Ortiz, J. Cioslowski and D. J. Fox, Gaussian 09, Revision E.01; Gaussian, Inc.:Wallingford CT, 2010.

[4] S. Wang, W. Yao, J. Lin, Z. Ding and X. Wang, Angew. Chem., Int. Ed., 2014, 53,1034

[5] K. M. Choi, D. Kim, B. Rungtaweevoranit, C. A. Trickett, J. T. Barmanbek, A. S. Alshammari, P. Yang and O. M. Yaghi, J. Am. Chem. Soc., 2017, 139, 356.

[6] R. Huang, Y. Peng, C. Wang, Z. Shi and W. Lin, Eur. J. Inorg. Chem., 2016, 4358.

[7] W. Zhu, C. Zhang, Q. Li, L. Xiong, R. Chen, X. Wan, Z. Wang, W. Chen, Z. Deng and Y. Peng, Appl. Catal. B: Environ, 2018, 238, 339.

[8] K. Niu, Y. Xu, H. Wang, R. Ye, H. L. Xin, F. Lin, C. Tian, Y. Lum, K. C. Bustillo, M. M. Doeff, M. T. M. Koper, J. Ager, R. Xu and H. Zheng, Sci Adv, 2017, 3, 1700921.

[9] S. Yang, W. Hu, X. Zhang, P. He, B. Pattengale, C. Liu, M. Cendejas, I. Hermans, X. Zhang, J. Zhang and J. Huang, J. Am. Chem. Soc., 2018, 140, 14614.

[10]Y. Wang, N. Y. Huang, J. Q. Shen, P. Q. Liao, X. M. Chen and J. P. Zhang, J. Am. Chem. Soc., 2018, 140, 38.

[11]C. Gao, S. Chen, Y. Wang, J. Wang, X. Zheng, J. Zhu, L. Song, W. Zhang and Y. Xiong, Adv. Mater., 2018, 30, 1704624.

[12]B. Han, X. Ou, Z. Deng, Y. Song, C. Tian, H. Deng, Y. J. Xu and Z. Lin, Angew. Chem., Int. Ed., 2018, 57, 16811.

[13]C. Gao, Q. Meng, K. Zhao, H. Yin, D. Wang, J. Guo, S. Zhao, L. Chang, M. He, 
Q. Li, H. Zhao, X. Huang, Y. Gao and Z. Tang, Adv. Mater., 2016, 28, 6485.

[14]T. Kajiwara, M. Fujii, M. Tsujimoto, K. Kobayashi, M. Higuchi, K. Tanaka and S. Kitagawa, Angew. Chem., Int. Ed., 2016, 55, 2697. 